\title{
Detached white dwarf main-sequence star binaries
}

\author{
B. Willems ${ }^{\star}$ and U. Kolb
}

\author{
Department of Physics and Astronomy, The Open University, Walton Hall, Milton Keynes, MK7 6AA, UK \\ e-mail: U.C.Kolb@open.ac.uk
}

Received 17 July 2003 / Accepted 27 February 2004

\begin{abstract}
We initiated a comprehensive state of the art binary population synthesis study of white dwarf main-sequence star (WDMS) binaries to serve as a foundation for subsequent studies on pre-cataclysmic variables, double white dwarfs, and white dwarf + B-star binaries. We considered seven distinct formation channels subdivided into three main groups according to the evolutionary process that gives rise to the formation of the white dwarf or its helium-star progenitor: dynamically stable Rochelobe overflow (Algol-type evolution), dynamically unstable Roche-lobe overflow (common-envelope evolution), or stellar winds (single star evolution). For each formation channel, we examine the sensitivity of the population to changes in the amount of mass lost from the system during dynamically stable Roche-lobe overflow, the common-envelope ejection efficiency, and the initial mass ratio or initial secondary mass distribution. In the case of a flat initial mass ratio distribution, the local space density of WDMS binaries is of the order of $\sim 10^{-3} \mathrm{pc}^{-3}$. This number decreases to $\sim 10^{-4} \mathrm{pc}^{-3}$ when the initial mass ratio distribution is approximately proportional to the inverse of the initial mass ratio. More than $75 \%$ of the WDMS binary population originates from wide systems in which both components essentially evolve as if they were single stars. The remaining part of the population is dominated by systems in which the white dwarf is formed in a common-envelope phase when the primary ascends the first giant branch or the asymptotic giant branch. When dynamically stable mass transfer proceeds highly conservative and the common-envelope ejection process is very efficient, the birthrate of WDMS binaries forming through a common-envelope phase is about 10 times larger than the birthrate of WDMS binaries forming through a stable Roche-lobe overflow phase. The ratio of the number of helium white dwarf systems to the number of carbon/oxygen or oxygen/neon/magnesium white dwarf systems derived from large samples of observed WDMS binaries by, e.g., future planet-search missions such as SuperWASP, COROT, and Kepler may furthermore constrain the common-envelope ejection efficiency.
\end{abstract}

Key words. stars: binaries: general - stars: evolution - stars: white dwarfs - methods: statistical

\section{Introduction}

In recent years, substantial numbers of detached white dwarf main-sequence star (WDMS) binaries have been detected in large-scale surveys searching for pre-cataclysmic variables (Hillwig et al. 2000; Raymond et al. 2003), double degenerates (Saffer et al. 1998), low-mass white dwarfs (Marsh et al. 1995; Maxted et al. 2000), planetary nebulae (Bond \& Livio 1990; Livio 1992), or even the dark matter content of the Galaxy's heavy halo (Silvestri et al. 2002). Upcoming surveys looking for transiting extrasolar planets are furthermore expected to contribute further to this rapidly growing database (Farmer \& Agol 2003). So far, the majority of WDMS binaries found consist of a white dwarf with a low-mass companion. White dwarfs in binaries with main-sequence secondaries more massive than $0.3 M_{\odot}$ are generally harder to detect due to the large luminosity difference between the component stars

Send offprint requests to: $\mathrm{B}$. Willems, e-mail: b-willems@northwestern.edu

* Present address: Northwestern University, Department of Physics and Astronomy, 2145 Sheridan Road, Evanston, IL 60208, USA.
(Iben et al. 1997; Marsh 2000). Despite this obstacle, several claims have recently been made on the possible detection of white dwarf binaries with bright companions of spectral types as early as B(e) (e.g. Holberg et al. 1998; Vennes et al. 1998; Burleigh \& Barstow 2000; Burleigh et al. 2001; Shobbrook et al. 2003, and references therein).

WDMS binaries with early $\mathrm{B}(\mathrm{e})$-type companions more massive than $\sim 10 M_{\odot}$ are thought to be the end product of binaries in which a giant-type star transfers mass to its initially less massive companion. The mass-transfer phase is responsible for exposing the giant's core as a white dwarf and for increasing the mass of the secondary so that it evolves towards earlier spectral types. In addition, transport of angular momentum may spin the secondary up to form a rapidly rotating Be star (Waters et al. 1988; Pols et al. 1991). The predicted number of WDMS binaries forming in this way is, however, rather small (e.g. de Kool \& Ritter 1993, Figs. 2 and 4) which may pose a problem if the recent claims on the detection of white dwarf + $\mathrm{B}$-star binaries are confirmed. In addition, as mass transfer is expected to increase the orbital period it is hard to explain the formation of short-period white dwarf + early B-star candidates 
such as HD $161103\left(P_{\text {orb }}=4.7\right.$ days, Shobbrook et al. 2003) through this evolutionary channel.

The wealth of existing and expected new data combined with new up-to-date stellar evolution tools (see Hurley et al. 2000) render the WDMS binaries a potentially fruitful subject of a comprehensive binary population synthesis study. A systematic exploration of the formation channels in particular may shed new insights on the origin of short-period white dwarf + B-star binaries. Other interesting subclasses of detached WDMS binaries include pre-cataclysmic variables and progenitors of double degenerates.

To the best of our knowledge, de Kool \& Ritter (1993) were the first to devote an entire study to the population of WDMS binaries as a class on its own. The authors used a Monte Carlo type simulation in which the initial binary parameters were randomly generated from observed orbital period, primary mass, and secondary mass distributions; and the evolution of the binaries was approximated by analytical fits to detailed stellar evolution tracks as described by Politano (1988) and de Kool (1992). The method resulted in distribution functions for the formation rates and space densities of detached WDMS binaries (or their direct progenitors) as a function of their expected orbital and stellar parameters.

In this paper, our aim is to update the population synthesis study by de Kool \& Ritter (1993) by using the more recent and much more detailed analytical fits to stellar evolution derived by Hurley et al. (2000). We study in more detail the differences between the evolutionary channels leading to the formation of WDMS binaries, and derive the formation rates and absolute numbers of systems for each of the formation channels separately. In addition, we consider a much more elaborate set of population synthesis models characterised by different assumptions about the fate of the mass transferred during dynamically stable Roche-lobe overflow, different common-envelope ejection efficiencies, and different initial mass ratio or secondary mass distributions.

Our method furthermore differs from that by de Kool \& Ritter (1993) in the implementation of the observed distributions of the initial masses and orbital periods: instead of randomly generating them from the observed distribution functions at the onset of the binary evolution calculations, we start our simulations from a logarithmically spaced 3-dimensional grid of initial masses and orbital periods in which each set of binary parameters has an equal probability. The likelihood of the different initial binary parameters is then taken into account by weighting the contribution of each WDMS binary found in the simulations according to the adopted primary mass distribution, initial mass ratio or secondary mass distribution, and initial orbital period distribution. In doing so, we obtain a better and more uniform sampling of the entire parameter space, which particularly benefits the resolution of low-density tails in the distribution functions describing the masses and orbital periods of WDMS binary components. This procedure also allows us to vary the distribution functions for the initial masses and orbital periods without having to repeat the binary evolution calculations.

The results of this study are intended to serve as a foundation for more focused population synthesis studies of white dwarf + B-star binaries (Willems et al., in preparation), double white dwarfs (Willems et al., in preparation), and precataclysmic variables. Hence, we here concentrate on the effect and the uncertainties associated with the different model parameters in general. In subsequent papers we hope to constrain these uncertainties by comparing our results with different types of WDMS binaries and WDMS binary descendants. The results presented here therefore do not necessarily represent the best possible fit to observationally derived binary parameters.

The plan of the paper is as follows. In Sect. 2, we present the basic ingredients and the assumptions adopted in the binary population synthesis calculations. In Sect. 3, we describe the different evolutionary channels leading to the formation of WDMS binaries as well as the ranges of stellar masses and orbital periods available to each formation channel. Section 4 deals with the effects of varying degrees of non-conservative mass transfer and different common-envelope ejection efficiencies on the population of WDMS binaries. In Sect. 5, we estimate the order of magnitude of the formation rates and the expected number of WDMS binaries currently populating the Galaxy. Section 6 briefly addresses the expected luminosity differences between the white dwarf and its companion, as well as their expected orbital radial-velocity variations. A brief summary of our results and some concluding remarks are presented in Sect. 7.

\section{Basic concepts and assumptions}

\subsection{The population synthesis code}

We use the BiSEPS binary population synthesis code introduced by Willems \& Kolb (2002) to study the formation of detached white dwarf main-sequence star binaries. The code uses the single star evolution formulae derived by Hurley et al. (2000) and follows the main steps of the binary evolution scheme outlined by Hurley et al. (2002). All binary orbits are treated as circular and stellar rotation rates are kept synchronised with the orbital motion at all times. For the purpose of this investigation, we furthermore limit ourselves to Population I stellar compositions.

When a binary component overflows its Roche-lobe, the stability of the ensuing mass-transfer phase is determined by means of the radius-mass exponents introduced by Webbink (1985). Mass transfer taking place on the dynamical time scale of the donor star is assumed to lead to a common-envelope (CE) phase during which the orbital separation is reduced and the envelope is expelled from the system. The phase is modelled in the usual way by equating the binding energy of the donor star's envelope to the change in the orbital energy of the binary components. The orbital separation $a_{\mathrm{f}}$ at the end of the common-envelope phase is then related to the orbital separation $a_{\mathrm{i}}$ at the beginning of the phase as

$\frac{a_{\mathrm{f}}}{a_{\mathrm{i}}}=\frac{M_{\mathrm{c}} / M_{1}}{1+2 M_{\mathrm{e}} /\left(M_{2} \alpha_{\mathrm{CE}} \lambda r_{\mathrm{L}}\right)}$.

Here $G$ is the gravitational constant, $M_{\mathrm{c}}$ and $M_{\mathrm{e}}$ are the core and envelope mass of the Roche-lobe overflowing star, 
$M_{1}=M_{\mathrm{c}}+M_{\mathrm{e}}$ is the total mass of the donor star, $M_{2}$ is the mass of the companion, and $r_{\mathrm{L}}=R_{\mathrm{L}} / a_{\mathrm{i}}$ is the radius of the donor star's Roche lobe in units of the orbital separation at the start of the common-envelope phase. For the bindingenergy parameter $\lambda$ and the common-envelope ejection efficiency $\alpha_{\mathrm{CE}}$, we adopt the commonly used values $\lambda=0.5$ and $\alpha_{\mathrm{CE}}=1.0$ (e.g. de Kool 1992; Politano 1996).

In the case of dynamically stable mass transfer, a fraction $1-\gamma_{\mathrm{RLOF}}$ of the transferred mass is assumed to be accreted by the donor star's companion, while the remaining fraction $\gamma_{\mathrm{RLOF}}$ is lost from the system carrying away the specific orbital angular momentum of the companion. The amount of angular momentum carried away by matter leaving the system during non-conservative Roche-lobe overflow is, however, still an unresolved issue, so that this quantity is effectively a free parameter (see, e.g., the Appendix in Kolb et al. 2001).

For non-degenerate accretors, we set

$1-\gamma_{\mathrm{RLOF}}=\min \left(10 \frac{\tau_{\dot{M}}}{\tau_{\mathrm{HK}, \mathrm{a}}}, 1\right)$

where $\tau_{\dot{M}}$ is the mass-transfer time scale of the donor and $\tau_{\mathrm{HK}, \mathrm{a}}$ is the thermal time scale of the accretor. With this prescription, the mass-transfer phase is conservative as long as $\tau_{\mathrm{HK}, \mathrm{a}}<$ $10 \tau_{\dot{M}}$ (see also, for example, Iben \& Tutukov 1987; Pols et al. 1991; Hurley et al. 2002). Accretion onto white dwarfs is assumed to be fully non-conservative so that $\gamma_{\mathrm{RLOF}}=1$ and $\dot{M}_{\mathrm{WD}}=0$, where $M_{\mathrm{WD}}$ is the mass of the white dwarf. This assumption does not affect the formation of detached WDMS binaries, but may influence the fate of their descendants when the main-sequence star becomes larger than its critical Roche lobe. We do not deal with neutron star and black hole accretors in this investigation.

For more details on the treatment of mass-loss and massaccretion in the BiSEPS code we refer to Willems \& Kolb (2002).

\subsection{Initial masses and orbital periods}

We start our population synthesis study by evolving a large number of binaries initially consisting of two zero-age mainsequence stars with a mass between 0.1 and $30 M_{\odot}$ and an orbital period between 0.1 and 100000 days. The initial primary and secondary masses, $M_{1}$ and $M_{2}$, and the initial orbital periods $P_{\text {orb }}$ are taken from a grid consisting of 60 logarithmically spaced stellar masses and 300 logarithmically spaced orbital periods. The maximum evolutionary age considered for each binary is $10 \mathrm{Gyr}$. For symmetry reasons only binaries with $M_{1}>M_{2}$ are evolved.

The number of systems following a sequence of evolutionary phases similar to those of a given binary in our simulated sample is determined by the probability of the binary's initial parameters, by the star formation rate at the birth of the zeroage main-sequence binary, and by the fraction of stars in binaries. We assume the initial primary masses to be distributed according to the normalised initial mass function (IMF) ${ }^{1}$

$$
\xi\left(M_{1}\right)= \begin{cases}0 & M_{1} / M_{\odot}<0.1 \\ 0.38415 M_{1}^{-1} & 0.1 \leq M_{1} / M_{\odot}<0.75 \\ 0.23556 M_{1}^{-2.7} & 0.75 \leq M_{1} / M_{\odot}<\infty\end{cases}
$$

the initial mass ratios $q=M_{2} / M_{1}$ according to

$n(q)= \begin{cases}\mu q^{v} & 0<q \leq 1, \\ 0 & q>1,\end{cases}$

and the initial orbital separations $a$ according to

$$
\chi(a)= \begin{cases}0 & a / R_{\odot}<3 \text { or } a / R_{\odot}>10^{6}, \\ 0.078636 a^{-1} 3 \leq a / R_{\odot} \leq 10^{6} .\end{cases}
$$

In Eq. (4), $v$ is a constant and $\mu$ a normalisation factor depending on $v$. Unless stated otherwise, we set $\nu=0$ and $\mu=1$. The upper limit of $10^{6} R_{\odot}$ in the distribution of the initial orbital separations is larger than in our previous investigations in order to properly take into account the contribution of very wide systems to the population of WDMS binaries. For more details and references on the adopted distribution functions, we refer to Willems \& Kolb (2002).

We furthermore assume all stars to be in binaries and adopt a constant star-formation rate $S$ calibrated so that one binary with $M_{1}>0.8 M_{\odot}$ is born in the Galaxy each year (see also Iben \& Tutukov 1984; Han et al. 1995; Hurley et al. 2002). When combined with an effective Galactic volume of $5 \times$ $10^{11} \mathrm{pc}^{3}$, this yields an average local birthrate of Galactic white dwarfs of $2 \times 10^{-12} \mathrm{pc}^{-3} \mathrm{yr}^{-1}$, which is consistent with observations (Weidemann 1990). From this calibration, it follows that

$S \int_{0.8}^{\infty} \xi\left(M_{1}\right) \mathrm{d} M_{1}=1$,

so that $S=4.9 \mathrm{yr}^{-1}$. This rate may be converted into an approximate local star formation rate (expressed in $\mathrm{pc}^{-3} \mathrm{yr}^{-1}$ ) by dividing it by $5 \times 10^{11} \mathrm{pc}^{3}$. As the star formation rate may have been higher in the past, the calibration of the rate to match the observationally inferred current birthrate of Galactic white dwarfs may yield an underestimate of the number of binaries with old component stars (Boissier \& Prantzos 1999).

\section{Formation channels}

Stars in close binaries can evolve into white dwarfs either through the loss of their envelope by the action of a stellar wind or by mass transfer resulting from dynamically stable or unstable Roche-lobe overflow. For brevity, we refer to binaries that do not undergo mass-transfer episodes as non-interacting binaries, even if some mass exchange and orbital evolution takes

1 We note that the adopted IMF is a simplified version of the IMF by Kroupa et al. (1993). The simplification is introduced because of the still existing uncertainties in the IMF for low-mass stars (see, e.g., Scalo 1998; Kroupa 2001). Its effect on our results is small in comparison to the overall uncertainties of the population synthesis models. 
place due to the action of a stellar wind. Binaries in which a white dwarf or its direct progenitor is formed as the end product of mass transfer are referred to as interacting binaries. In what follows, we divide the latter group according to the stability of the mass-transfer phase and according to the remnant it leaves behind.

Since detached WDMS binaries constitute an important intermediary phase in the formation of many more exotic binary systems such as cataclysmic variables and double degenerates, some of the formation channels described below have been partially discussed before, albeit maybe in lesser detail and in different contexts. The evolution of binaries with low- to intermediate-mass component stars has been studied in detail by, e.g., Iben \& Tutukov (1985, 1986a, 1987), van der Linden (1987), de Loore \& Vanbeveren (1995), Langer et al. (2000), Han et al. (2000), Nelson \& Eggleton (2001), Chen \& Han (2002, 2003); and references therein.

\subsection{Dynamically stable mass transfer}

\subsubsection{Case B RLOF with a white dwarf remnant}

The first formation channel applies to initial binaries consisting of two low-mass main-sequence stars with orbital periods that are too short to allow the primary to evolve on the giant branch without overflowing its Roche lobe. Most of the systems initiate mass transfer on the thermal time scale of the donor star when it approaches the end of the main sequence or when it crosses the Hertzsprung gap. Once the mass ratio is inverted, mass transfer generally slows down and continues as the donor star ascends the first giant branch. In most cases, the case B mass-transfer phase is more important for determining the subsequent evolution than the initial case A phase. For brevity, we therefore simply refer to the case $\mathrm{B}$ phase as the phase characterising the formation channel.

Since the thermal time scale of the accreting companion is of the same order of magnitude as that of the donor star, the mass-transfer phase is highly conservative (see Eq. (2)). The secondary's mass and the orbital period may therefore increase substantially with respect to their values at the onset of the Roche-lobe overflow phase. The mass-transfer phase terminates when the giant's entire envelope is transferred to the companion, exposing its helium core as a low-mass white dwarf. The main evolutionary phases characterising this formation channel are summarised schematically in Fig. 1. For future reference, we label this channel as channel 1.

The two-dimensional probability distribution functions (PDFs) describing the regions of the $\left(M_{1}, P_{\text {orb }}\right)$ - and the $\left(M_{2}, P_{\text {orb }}\right)$-planes occupied by binaries evolving through formation channel 1 are displayed in the upper panels of Figs. 2 and 3. The left-hand panels of the figures show the distributions of the WDMS binary progenitors at the beginning of their evolution as zero-age main-sequence binaries, while the righthand panels show the distributions of the WDMS binaries at the time of their formation. For the construction of the plots, only binaries contributing to the present-day Galactic population are

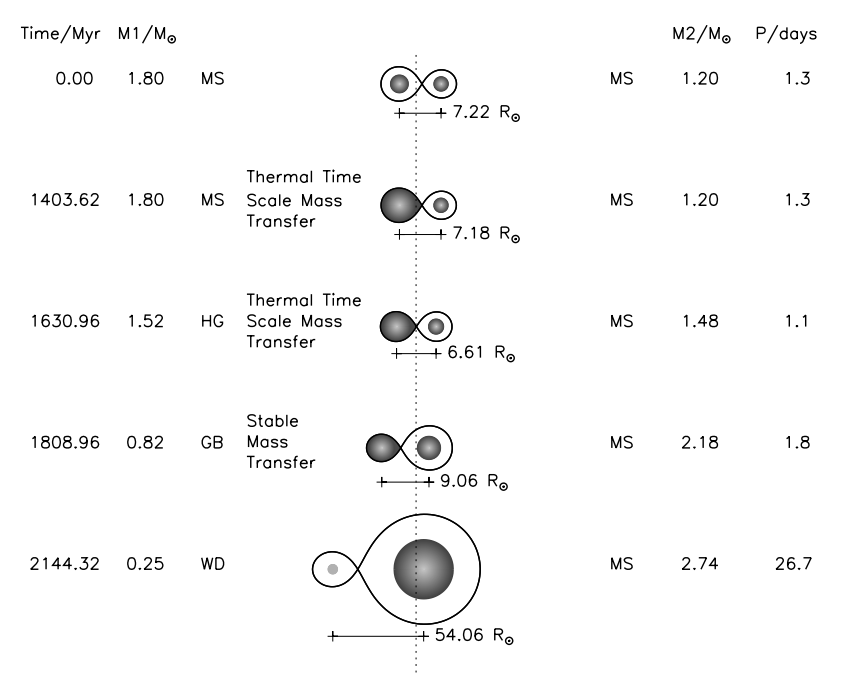

Fig. 1. Schematic representation of the main evolutionary phases leading to the formation of a WDMS binary via evolutionary channel 1 (see text for details). MS stands for main sequence, HG for Hertzsprung gap, GB for giant branch, and WD for white dwarf.

taken into account ${ }^{2}$. The distributions are normalised so that the bin containing the largest contribution to the population has a PDF value equal to one. In order to show the maximum amount of detail this normalisation is performed separately for each of the formation channels considered. The relative importance of the different formation channels will be illustrated in Sect. 3.4 and addressed in more detail in Sect. 5.

The initial orbital periods of the binaries evolving through formation channel 1 typically range from 0.5 to 3 days. Binaries with shorter orbital periods tend to converge before the primary is able to evolve into a white dwarf due to the angular momentum losses caused by magnetic braking and/or gravitational radiation (Pylyser \& Savonije 1988, 1989). Systems with longer orbital periods on the other hand have somewhat too evolved donor stars to initiate a dynamically stable Roche-lobe overflow phase. Instead, they undergo a commonenvelope phase which, in view of the short initial periods, generally results in the merger of the donor star's core with its main-sequence companion.

The initial primary and secondary masses available to formation channel 1 are limited to the intervals given by $1 M_{\odot} \lesssim$ $M_{1} \lesssim 5 M_{\odot}$ and $M_{2} \lesssim 3 M_{\odot}$. The primary mass interval arises from the dual requirement that the primary must be massive enough to evolve away from the zero-age main sequence within the imposed age limit of $10 \mathrm{Gyr}$, but not so massive that its core mass at the end of the mass-transfer phase is high

\footnotetext{
2 Note that our purpose here is to examine the formation space of WDMS binaries. The plots are therefore not fully representative for the present-day population of short-period systems $\left(P_{\text {orb }} \lesssim\right.$ 1 day) which may have undergone significant orbital shrinkage due to magnetic braking and/or gravitational radiation after their formation. However, even though the orbital evolution is not shown in the figures, the finite life time of the systems resulting from the orbital evolution and/or the nuclear evolution of the secondary is taken into account in the determination of the WDMS binaries currently populating the Galaxy.
} 

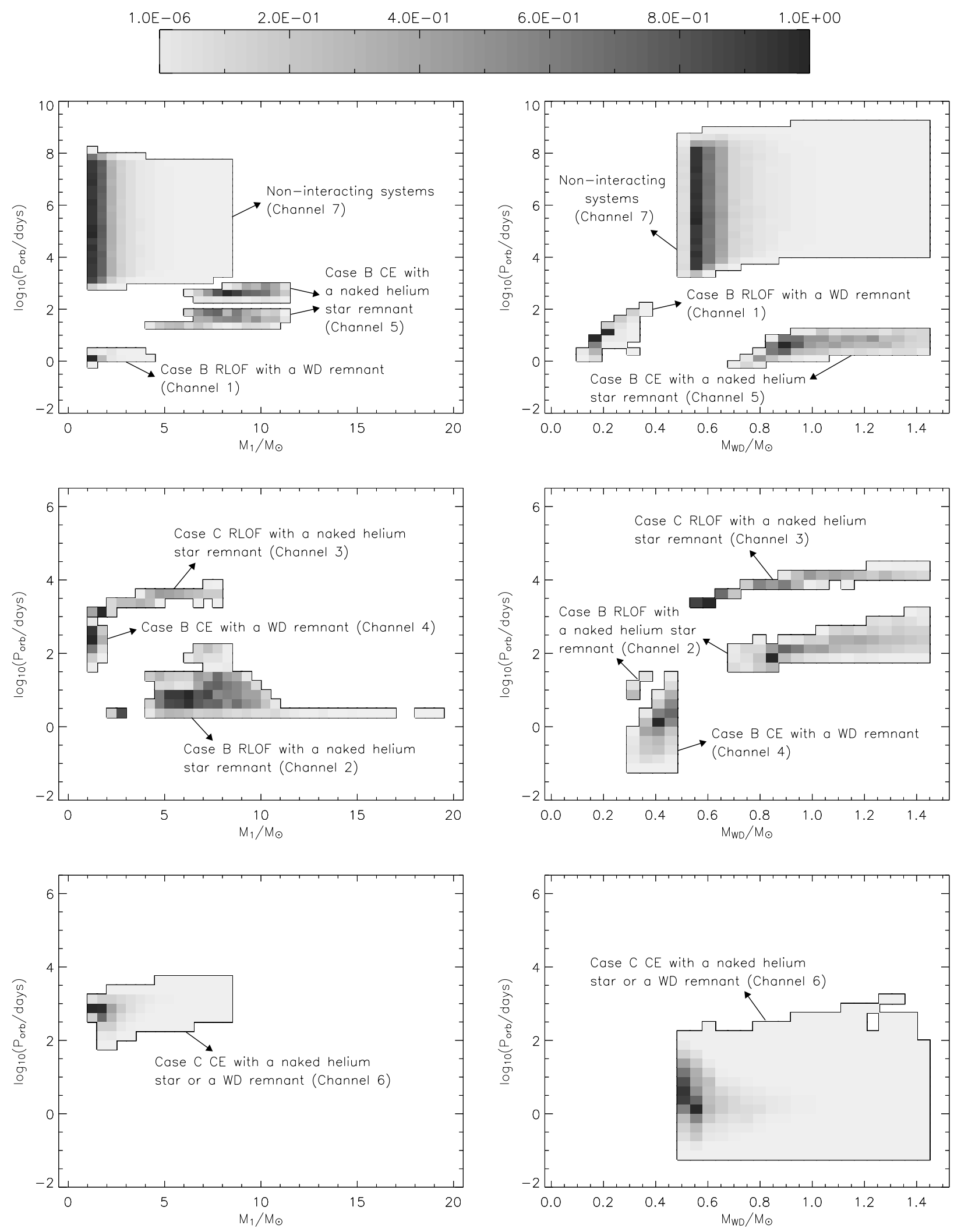

Fig. 2. Distribution of present-day WDMS binaries in the $\left(M_{1}, P_{\text {orb }}\right)$-plane at the beginning of the progenitors' evolution (left-hand panels) and at the beginning of the WDMS binary phase (right-hand panels). The distributions are normalised so that for each formation channel the bin containing the largest contribution to the population has a PDF value equal to one.

enough to ignite helium in its central layers. In our model, stars more massive than $\sim 2.5 M_{\odot}$ are still able to evolve into helium white dwarfs due to the reduction in mass caused by the thermal time scale mass-transfer phase on the main sequence. 

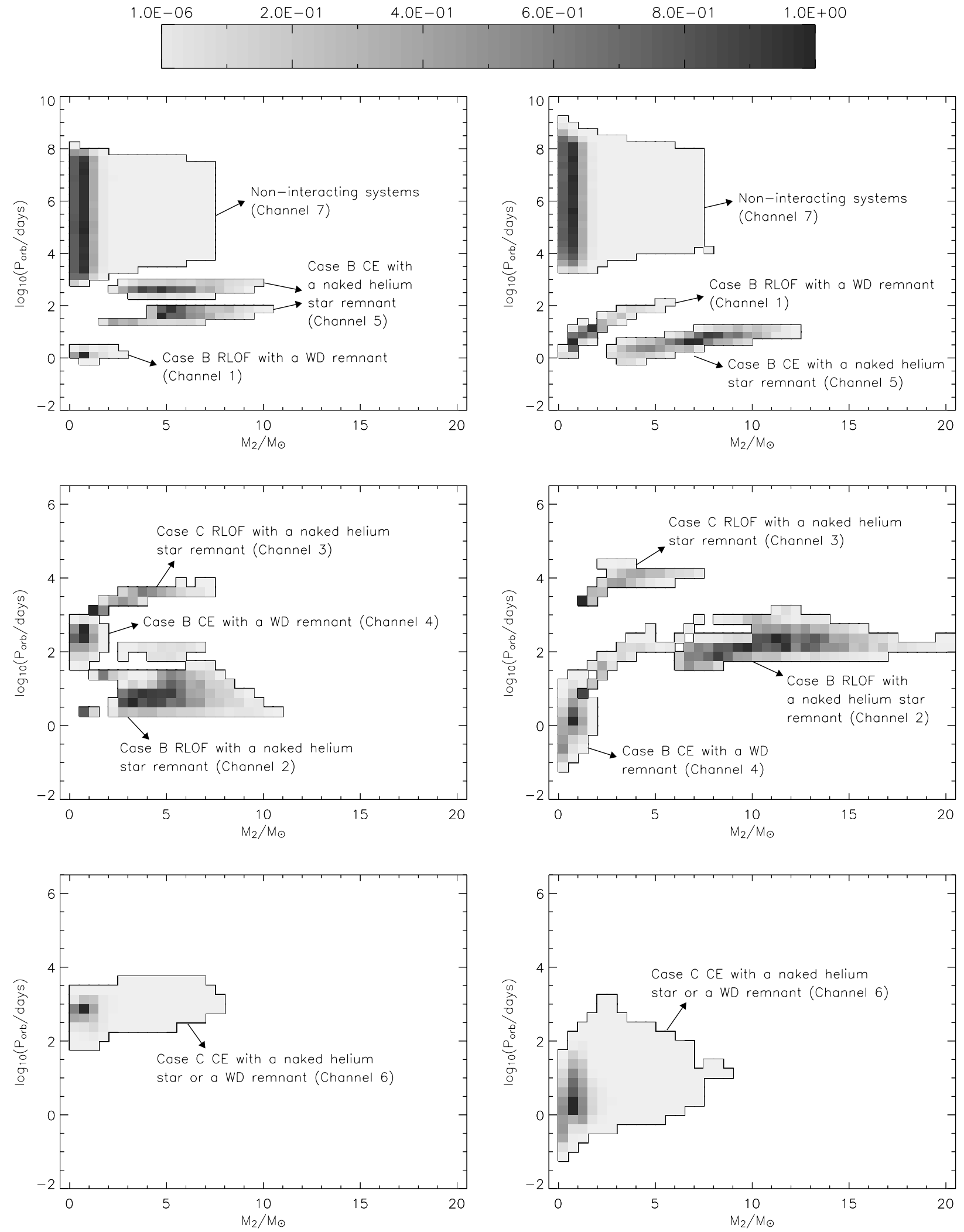

Fig. 3. Distribution of present-day WDMS binaries in the $\left(M_{2}, P_{\text {orb }}\right)$-plane at the beginning of the progenitors' evolution (left-hand panels) and at the beginning of the WDMS binary phase (right-hand panels). The distributions are normalised in the same way as in Fig. 2.

The upper limit of $3 M_{\odot}$ on the secondary mass is imposed by the secondary's main-sequence life time which needs to be long enough to allow the primary to evolve into a white dwarf before the secondary leaves the main sequence. Since the life time of a star on the main sequence decreases with increasing values of its mass, conservative mass transfer here yields 
a competitive race between the formation of the white dwarf and the accelerating evolution of the secondary. A similar behaviour can be deduced from the evolutionary scenarios described by Iben \& Tutukov (1985).

The formation channel eventually gives rise to WDMS binaries consisting of a $0.1-0.4 M_{\odot}$ He white dwarf and a mainsequence star with a mass up to $6 M_{\odot}$. Due to the stable masstransfer phase on the giant branch the orbital periods may be as long as 100 days. The majority of the newly formed WDMS binaries have an orbital period which is correlated with the mass of the white dwarf. The correlation arises during the masstransfer phase on the giant branch where the radius of the giant, which is approximately equal to the radius of its Roche-lobe, is determined by the mass of its core. The same relation gives rise to the well-known correlation between the white dwarf mass and the orbital period in wide binary millisecond pulsars (see, e.g., Joss et al. 1987; Savonije 1987; Rappaport et al. 1995; Ritter 1999; Tauris \& Savonije 1999). The small number of WDMS binaries occupying the region below the $P_{\text {orb }}-M_{\mathrm{WD}}$ relation correspond to thermal time scale mass-transfer systems for which the primary already lost most its envelope prior to the stable mass-transfer phase on the giant branch. The orbital periods of the newly formed WDMS binaries furthermore increase with increasing mass of the secondary. This relation arises from the narrow range of initial orbital periods and secondary masses available to the formation channel and from the increase of the orbital period with the amount of mass transferred during the conservative Roche-lobe overflow phase on the giant branch.

\subsubsection{Case B RLOF with a naked helium star remnant}

Binaries evolving through the second formation channel start their evolution with more massive component stars and somewhat longer orbital periods than those in channel 1 . They again undergo a highly conservative case B mass-transfer phase, possibly preceded by a short case A phase during the final stages of the primary's evolution on the main sequence. Since the initial primary masses are higher and the initial orbital periods are longer than in channel 1, the core of the Roche-lobe filling giant is now massive enough to ignite helium in its central layers so that a naked helium star is formed instead of a white dwarf. During the subsequent evolution, the naked helium star at some point loses its envelope either through the action of a stellar wind or through a short second phase of stable Roche-lobe overflow. The outcome in both cases is a relatively wide binary consisting of a $\mathrm{C} / \mathrm{O}$ or $\mathrm{O} / \mathrm{Ne} / \mathrm{Mg}$ white dwarf and an intermediate- to high-mass main-sequence secondary. The main evolutionary phases of this formation channel are summarised schematically in Fig. 4. We will refer to the channel as channel 2.

From the middle panels of Figs. 2 and 3, it follows that most of the WDMS binaries evolving through formation channel 2 originate from binaries with initial orbital periods between 2 and 130 days, initial primary masses between $2 M_{\odot}$ and $12 M_{\odot}$, and initial secondary masses smaller than $11 M_{\odot}$. We note that there is a small number of systems with primary masses up to $20 M_{\odot}$ for which highly conservative mass

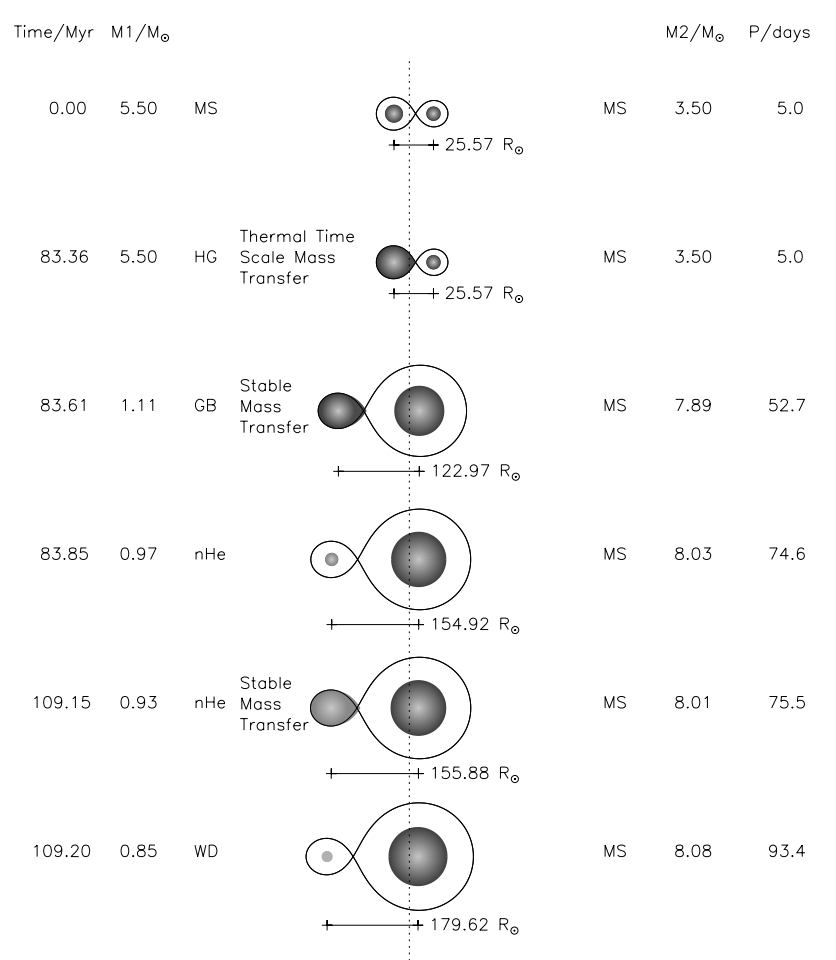

Fig. 4. As Fig. 1, but for formation channel 2. nHe stands for naked helium star.

transfer results in the formation of secondaries with masses up to $30 M_{\odot}$. However, since it is uncertain whether or not a white dwarf may be formed from primaries with such high initial masses, we do not include these systems in the following discussion.

The limits on the range of initial orbital periods result from the evolutionary stage of the primary at the onset of the first Roche-lobe overflow phase. For binaries with initial orbital periods shorter than 2 days, the core of the primary emerges from the mass-transfer phase as a low-mass naked helium star with a helium-burning life time that is too long for it to form a white dwarf before the secondary leaves the main sequence. Binaries with initial orbital periods longer than 130 days on the other hand have significantly evolved donor stars with deep convective envelopes. They are therefore subjected to a commonenvelope phase instead of to a thermal time scale mass-transfer phase.

The lower limit of $2 M_{\odot}$ on the mass of the primary stems from the requirement that the star must be able to develop a sufficiently massive core capable of igniting helium at the end of the stable mass-transfer phase on the giant branch. The upper limit of $12 M_{\odot}$ corresponds to the highest mass for which a star subjected to mass loss may evolve into a white dwarf rather than into a neutron star (e.g. van den Heuvel 1981; Law \& Ritter 1983). The lower and upper limits on the secondary mass result from the requirement that mass transfer from the primary is dynamically stable and from our convention that the secondary is initially less massive than the primary.

At the time of formation, the WDMS binaries forming through formation channel 2 typically consist of a 
1064

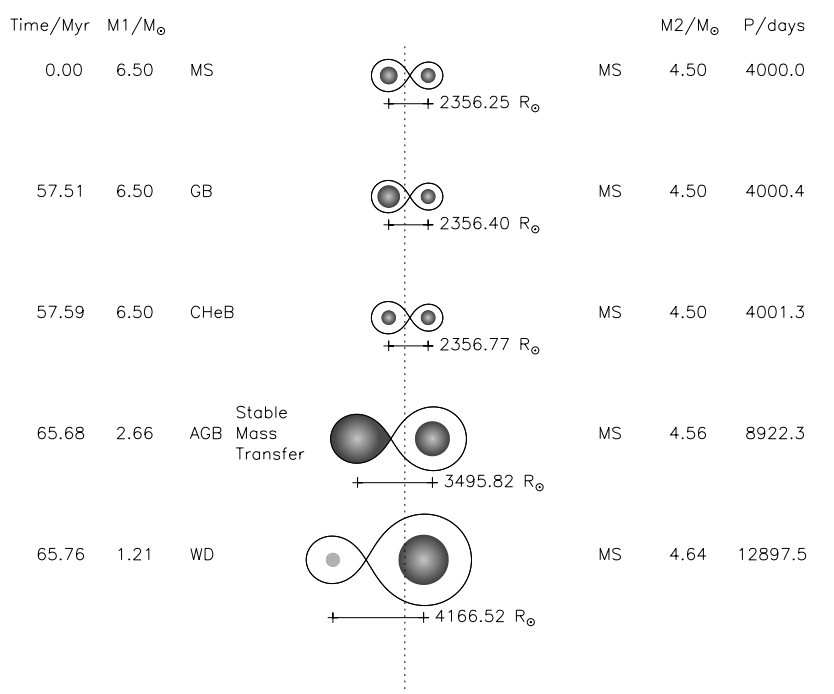

Fig. 5. As Fig. 1, but for formation channel 3. $\mathrm{CHeB}$ stands for core helium burning on the horizontal branch.

$0.65-1.44 M_{\odot} \mathrm{C} / \mathrm{O}$ or $\mathrm{O} / \mathrm{Ne} / \mathrm{Mg}$ white dwarf and a $1-20 M_{\odot}$ main-sequence star. The orbital periods range from 10 to 1000 days, with the bulk of the systems occupying a rather narrow band of orbital periods around $P_{\text {orb }} \approx 200$ days.

\subsubsection{Case C RLOF with a white dwarf remnant}

The third evolutionary channel applies to binaries with initial orbits wide enough to allow the primary to evolve to the bottom of the asymptotic giant branch (AGB) without overflowing its Roche lobe. As it ascends the AGB, a strong stellar wind decreases the primary's mass below $2 / 3$ of the mass of its companion so that when it finally does fill its Roche-lobe, the ensuing mass-transfer phase is dynamically stable (e.g. Webbink et al. 1983). When mass transfer ends, any remaining surface layers are quickly removed by the wind, leading to the exposure of the AGB star's $\mathrm{C} / \mathrm{O}$ or $\mathrm{O} / \mathrm{Ne} / \mathrm{Mg}$ core as a white dwarf. The orbital period of the newly formed WDMS binary may be substantially longer than the initial binary period due to the combined effect of the stellar wind and the stable mass-transfer phase on the AGB. The main evolutionary phases followed by a binary evolving through this formation channel are summarised schematically in Fig. 5. We will refer to the channel as formation channel 3.

The middle panels of Figs. 2 and 3 show that the binaries evolving through formation channel 3 have initial orbital periods between 600 and 10000 days. In binaries with shorter orbital periods the AGB wind has insufficient time to decrease the mass ratio below $2 / 3$ before the primary fills its Roche lobe. The resulting mass-transfer phase is then dynamically unstable and leads to the formation of a common-envelope (see Sect. 3.2.3). Binaries with initial orbital periods longer than 10000 days are too wide to interact.

The initial primary and secondary masses range from $1 M_{\odot}$ to $8 M_{\odot}$, similar to the mass range leading to the formation of white dwarfs by single stars. This conformity arises because the primary's Roche-lobe overflow phase does not take place

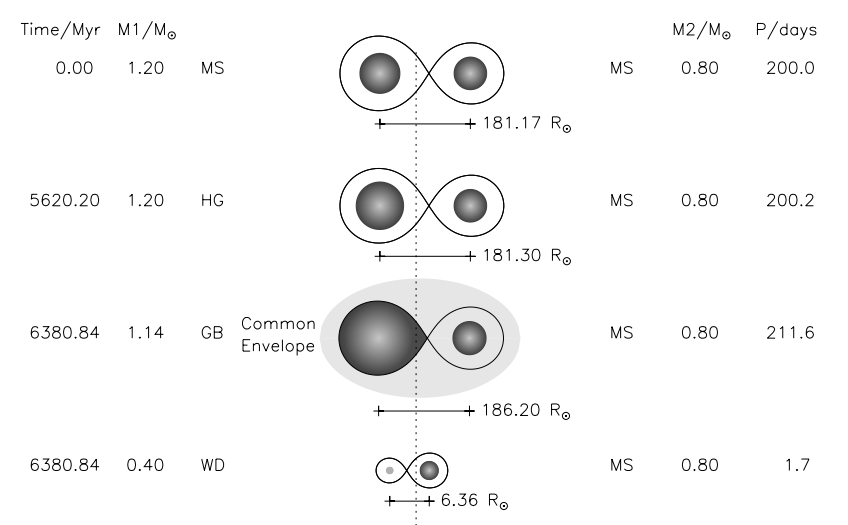

Fig. 6. As Fig. 1, but for formation channel 4.

until the very late stages of the AGB (see also Iben \& Tutukov 1985). The fact that the mass ratio needs to drop below $2 / 3$ furthermore implies that the initial secondary mass generally cannot be too much smaller than the initial primary mass.

The formation channel eventually gives rise to WDMS binaries consisting of a $0.5-1.44 M_{\odot} \mathrm{C} / \mathrm{O}$ or $\mathrm{O} / \mathrm{Ne} / \mathrm{Mg}$ white dwarf and a 1-8 $M_{\odot}$ main-sequence star revolving around each other with a period of 2000 to 20000 days.

\subsection{Dynamically unstable mass transfer}

\subsubsection{Case B CE phase with a white dwarf remnant}

The fourth evolutionary channel is characterised by a dynamically unstable mass-transfer phase from a low-mass giantbranch star. As the secondary plunges into the donor star's rapidly expanding envelope, the orbit shrinks and orbital energy is transferred to the envelope until it is expelled from the system. At the end of the phase, the core of the Roche-lobe filling giant is exposed as a helium white dwarf which orbits the main-sequence secondary with a drastically reduced orbital separation. The main evolutionary phases comprising this formation channel are summarised schematically in Fig. 6. We will refer to the channel as channel 4.

It follows from the middle panels of Figs. 2 and 3 that the systems evolving through formation channel 4 have initial orbital periods between 30 and 1000 days. Binaries with initial orbital periods shorter than 30 days do not survive the commonenvelope phase evoked by the primary, while binaries with orbital periods longer than 1000 days do not undergo Roche-lobe overflow until the primary reaches the AGB. The limits on the initial primary and secondary mass ranges, $1 M_{\odot} \lesssim M_{1} \lesssim 2 M_{\odot}$ and $M_{2} \lesssim 2 M_{\odot}$, arise for similar reasons as those in formation channel 1 (see Sect. 3.1.1). The upper limits on $M_{1}$ and $M_{2}$ are here somewhat smaller because the longer initial orbital periods allow a primary of a given mass to reach a more evolved evolutionary state and thus to develop a more massive helium core than the same primary in a shorter-period binary. The mass and period ranges of binaries surviving the common-envelope phase in this evolutionary channel are in excellent agreement with the three-dimensional hydrodynamical simulations performed by Sandquist et al. (2000). 


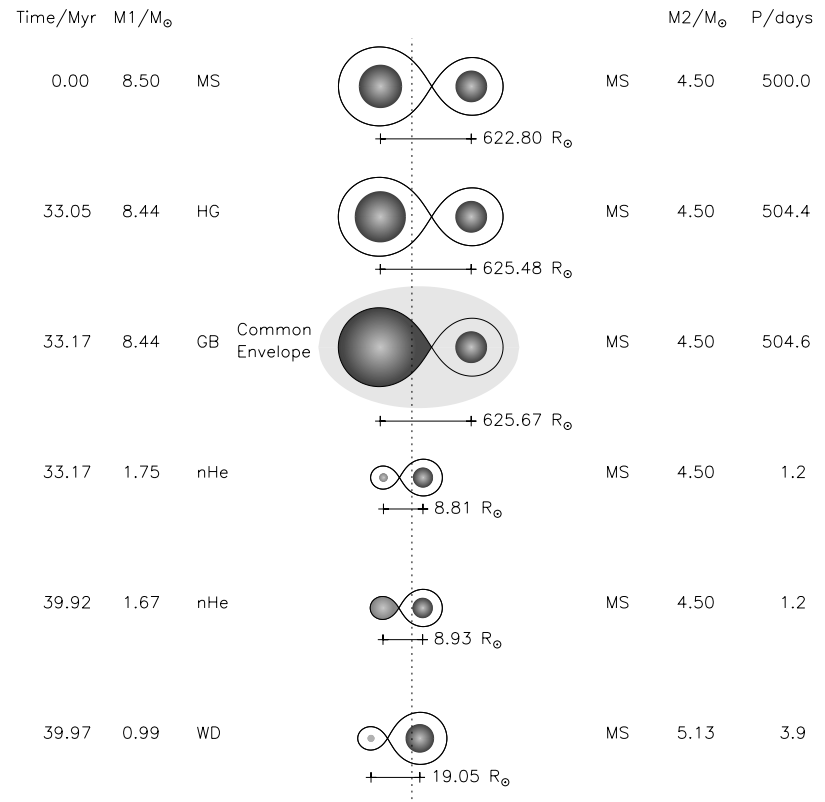

Fig. 7. As Fig. 1, but for formation channel 5.

The formation channel results in WDMS binaries consisting of a $0.3-0.5 M_{\odot}$ He white dwarf and a main-sequence star with a mass up to $2 M_{\odot}$. The orbital period takes values between 0.05 and 30 days. The majority of the systems have $P_{\text {orb }} \lesssim 3$ days, $M_{\mathrm{WD}} \approx 0.4 M_{\odot}$ and $M_{2} \lesssim 1 M_{\odot}$.

\subsubsection{Case B CE phase with a naked helium star remnant}

In the fifth evolutionary channel, a common-envelope phase occurs when an intermediate- to high-mass primary crosses the Hertzsprung-gap or ascends the first giant branch. The core of the primary emerges from the spiral-in phase as a lowmass naked helium star which orbits the main-sequence secondary with an orbital period of the order of a few days. The following few million years, the primary burns helium in its core until it loses its surface layers in a stellar wind or until it overflows its Roche lobe a second time when helium is exhausted in the core. In either case, the primary evolves into a $\mathrm{C} / \mathrm{O}$ or $\mathrm{O} / \mathrm{Ne} / \mathrm{Mg}$ white dwarf orbiting an intermediate- to high-mass main-sequence secondary. The main evolutionary phases characterising this formation channel are summarised schematically in Fig. 7. We will refer to this channel as formation channel 5 .

The upper panels of Figs. 2 and 3 show that the formation channel applies to binaries with initial orbital periods between 10 and 1000 days, initial primary masses between $4 M_{\odot}$ and $12 M_{\odot}$, and initial secondary masses between $1 M_{\odot}$ and $11 M_{\odot}$. Stars in binaries with orbital periods shorter then 10 days are so close that Roche-lobe overflow from the primary usually already occurs before it has developed a deep convective envelope, so that mass transfer tends to be dynamically stable (see formation channel 2, Sect. 3.1.2). Binaries with periods longer than 1000 days on the other hand allow the primary to evolve beyond the first giant branch without filling its Roche lobe. The gap in the initial period range around $\sim 100$ days separates systems initiating mass transfer in the Hertzsprung gap from those initiating mass transfer on the giant branch. Mass transfer from Hertzsprung-gap donor stars often starts of as a thermal time scale mass-transfer phase which evolves into a common-envelope phase as the donor star approaches the giant branch. The survival of these systems depends strongly on the ability of the thermal time scale mass-transfer phase to decrease the primary's mass sufficiently before the onset of the common-envelope phase. The gap between systems with Hertzsprung-gap and giant-branch donor stars is related to the behaviour of the adiabatic radius-mass exponents tabulated by Hjellming (1989). The large values found by Hjellming (1989) near the transition phase where the star starts to develop a deep convective envelope yield a small window in the parameter space where the mass-transfer phase is dynamically stable, so that a different evolutionary scenario ensues (e.g. formation channel 2). However, in view of the still existing uncertainties in the detailed modelling of this transition phase, the associated values of the adiabatic radius-mass exponents are also quite uncertain. The occurrence of the gap may therefore be an artifact of the stability criterion separating systems undergoing dynamically stable Roche-lobe overflow from those undergoing dynamically unstable Roche-lobe overflow. The origin of the limits on the primary mass range is similar to that of the limits found for formation channel 2 (Sect. 3.1.2). The lower limit of $1 M_{\odot}$ on the secondary mass corresponds to the smallest companion mass for which the binary is able to avoid a merger.

At the time of formation, the WDMS binaries forming through formation channel 5 consist of a $0.65-1.44 M_{\odot} \mathrm{C} / \mathrm{O}$ or $\mathrm{O} / \mathrm{Ne} / \mathrm{Mg}$ white dwarf and a $2-13 M_{\odot}$ main-sequence star orbiting each other with a period of 0.5 to 20 days. The high secondary masses result from mass accretion during the second Roche-lobe overflow phase of the primary.

\subsubsection{Case C CE phase with a naked helium star or a white dwarf remnant}

Similar to formation channel 3, the initial orbital separations of the binaries following the sixth evolutionary channel are wide enough to avoid any type of Roche-lobe overflow until the primary reaches the AGB. However, the primary here fills its critical Roche lobe before the wind has a chance to reduce the primary's mass below $2 / 3$ that of its companion so that the resulting mass-transfer phase is now dynamically unstable. The binary emerges from the ensuing common-envelope phase as a WDMS binary consisting of a $\mathrm{C} / \mathrm{O}$ or $\mathrm{O} / \mathrm{Ne} / \mathrm{Mg}$ white dwarf, the former core of the AGB primary, and a low- to intermediatemass main-sequence star. If the common-envelope phase takes place early on the $\mathrm{AGB}$, the $\mathrm{C} / \mathrm{O}$ or $\mathrm{O} / \mathrm{Ne} / \mathrm{Mg}$ core may retain a thin helium envelope which is subsequently stripped away by a Wolf-Rayet type stellar wind. The main evolutionary phases occurring in the formation channel are summarised schematically in Fig. 8. We will refer to this channel as formation channel 6. 
1066

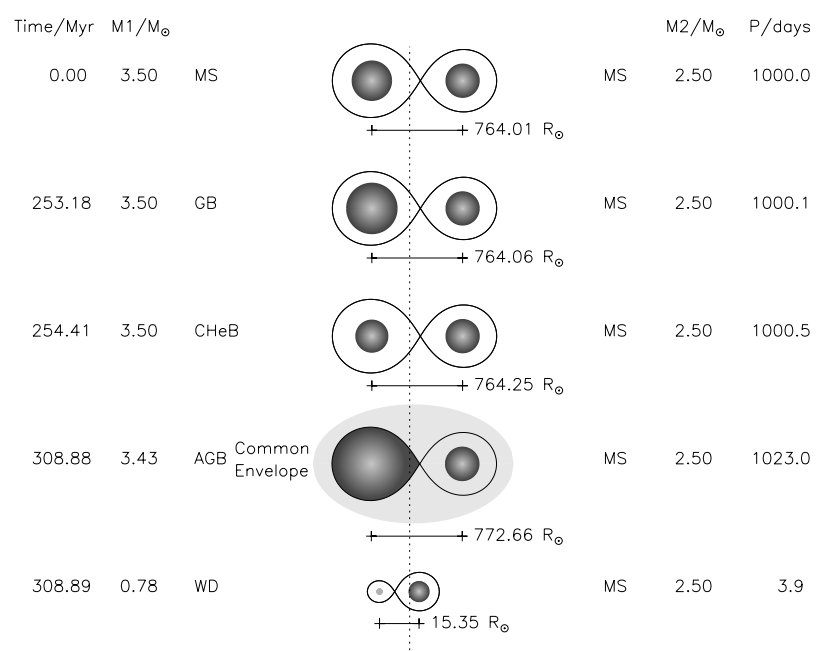

Fig. 8. As Fig. 1, but for formation channel 6.

From the bottom panels of Figs. 2 and 3 it follows that the systems evolving through formation channel 6 originate from binaries with initial orbital periods between 60 and 6500 days, initial primary masses between $1 M_{\odot}$ and $9 M_{\odot}$, and initial secondary masses smaller than or equal to $8 M_{\odot}$. The lower limit on the orbital period range stems from the requirement that the binaries survive the common-envelope phase, while the upper limit corresponds to the longest orbital period for which the primary fills its Roche lobe on the AGB. The limits on the primary and secondary masses arise for similar reasons as those in channel 3 (see Sect. 3.1.3).

The WDMS binaries forming through formation channel 6 typically consist of a $0.5-1.44 M_{\odot} \mathrm{C} / \mathrm{O}$ or $\mathrm{O} / \mathrm{Ne} / \mathrm{Mg}$ white dwarf and a main-sequence star with a mass up to $8 M_{\odot}$. The orbital period ranges from 0.05 to 2000 days, where the longperiod tail corresponds to systems in which the envelope mass of the primary is negligible in comparison to its core mass. A similar long-period tail was found by de Kool \& Ritter (1993, see their Fig. 2a). Most WDMS binaries forming through this channel have $M_{\mathrm{WD}} \approx 0.5-0.6 M_{\odot}, M_{2} \lesssim 2 M_{\odot}$, and $P_{\text {orb }} \lesssim$ 20 days.

\subsection{Non-interacting systems}

The last formation channel is the most straightforward one as it represents the non-interacting WDMS binaries. The initial orbital periods of these systems are wide enough for the two stars to evolve in much the same way as they would if they were single. Their contribution to the population is therefore independent of the assumptions adopted for the treatment of mass transfer in semi-detached binaries, so that they may provide a convenient means to renormalise our results for comparison with observations and with other authors.

The two-dimensional distribution functions describing the population of WDMS binaries forming without interacting are displayed in the upper panels of Figs. 2 and 3. The initial orbital periods are typically longer than 400 days, while the initial primary and secondary masses range from $1 M_{\odot}$ to $9 M_{\odot}$ and from $1 M_{\odot}$ to $8 M_{\odot}$, respectively. At the time of formation, most binaries have $M_{\mathrm{WD}} \approx 0.5-0.6 M_{\odot}$ and $M_{2} \lesssim 2 M_{\odot}$. The final orbital periods may be substantially longer than the initial ones due to the action the stellar wind responsible for exposing the primary's core as a $\mathrm{C} / \mathrm{O}$ or $\mathrm{O} / \mathrm{Ne} / \mathrm{Mg}$ white dwarf.

\subsection{The entire population}

In order to get an idea of the relative importance of the different formation channels, the formation space of all WDMS binaries forming through channels 1-7 is shown in Fig. 9 without renormalising the contributions of the different channels as in Figs. 2 and 3. The population is then clearly dominated by wide non-interacting systems with low-mass main-sequence stars evolving through formation channel 7 . The second largest group consists of systems with low-mass main sequence stars and periods in the range from 1 to 10 days originating from channels 4 and 6 . The dominance of WDMS binaries with wide non-interacting progenitors furthermore implies that the majority of the systems contain a $\mathrm{C} / \mathrm{O}$ white dwarf with a mass around $\sim 0.6 M_{\odot}$. Systems with white dwarf masses higher than $\sim 0.8 M_{\odot}$ or secondary masses higher than $2 M_{\odot}$ are relatively rare. This is further illustrated by the one-dimensional distribution functions for $M_{\mathrm{WD}}, M_{2}$, and $P_{\text {orb }}$ displayed in Fig. 10. The bimodal nature of the orbital period distribution is in good agreement with Fig. 5 of de Kool \& Ritter (1993).

We will quantify the relative contributions of the different formation channels in more detail in Sect. 5.

\section{The effect of $\gamma_{\text {RLOF }}$ and $\alpha_{\text {CE }}$}

The properties of the population of WDMS binaries forming through the evolutionary channels described in Sect. 3 may depend sensitively on the assumptions underlying the binary evolution calculations. The two main uncertainties applying to the discussed formation channels are the amount of mass accreted by a normal-type star during episodes of stable Roche-lobe overflow and the treatment of the common-envelope phase resulting from dynamically unstable Roche-lobe overflow. In order to assess how these uncertainties affect the WDMS binary population, we repeated the calculations presented in Sect. 3 for different mass-accretion parameters $\gamma_{\mathrm{RLOF}}$ and commonenvelope ejection efficiencies $\alpha_{\mathrm{CE}}$. The values of $\gamma_{\mathrm{RLOF}}$ and $\alpha_{\mathrm{CE}}$ adopted in the different population synthesis models are listed in Table 1. Our standard model (model A) corresponds to the assumptions described and used in Sects. 2 and 3.

The effects of $\gamma_{\mathrm{RLOF}}$ and $\alpha_{\mathrm{CE}}$ on the parameter space occupied by WDMS progenitors at the start of their evolution and by WDMS binaries at the time of their formation are summarised in Table 2. As in Figs. 2 and 3, only binaries contributing to the present-day Galactic population are taken into account. In order to focus on the bulk of the systems forming through each formation channel, the mass and period ranges quoted are limited to bins containing at least $\sim 1 \%$ of the channel's contribution to the WDMS binary population. In the case of model A, the extent of the parameter space listed in the table is therefore somewhat more restricted than in Sect. 3. We also note that by simply quoting the minimum and maximum values of $M_{1}, M_{2}$, 

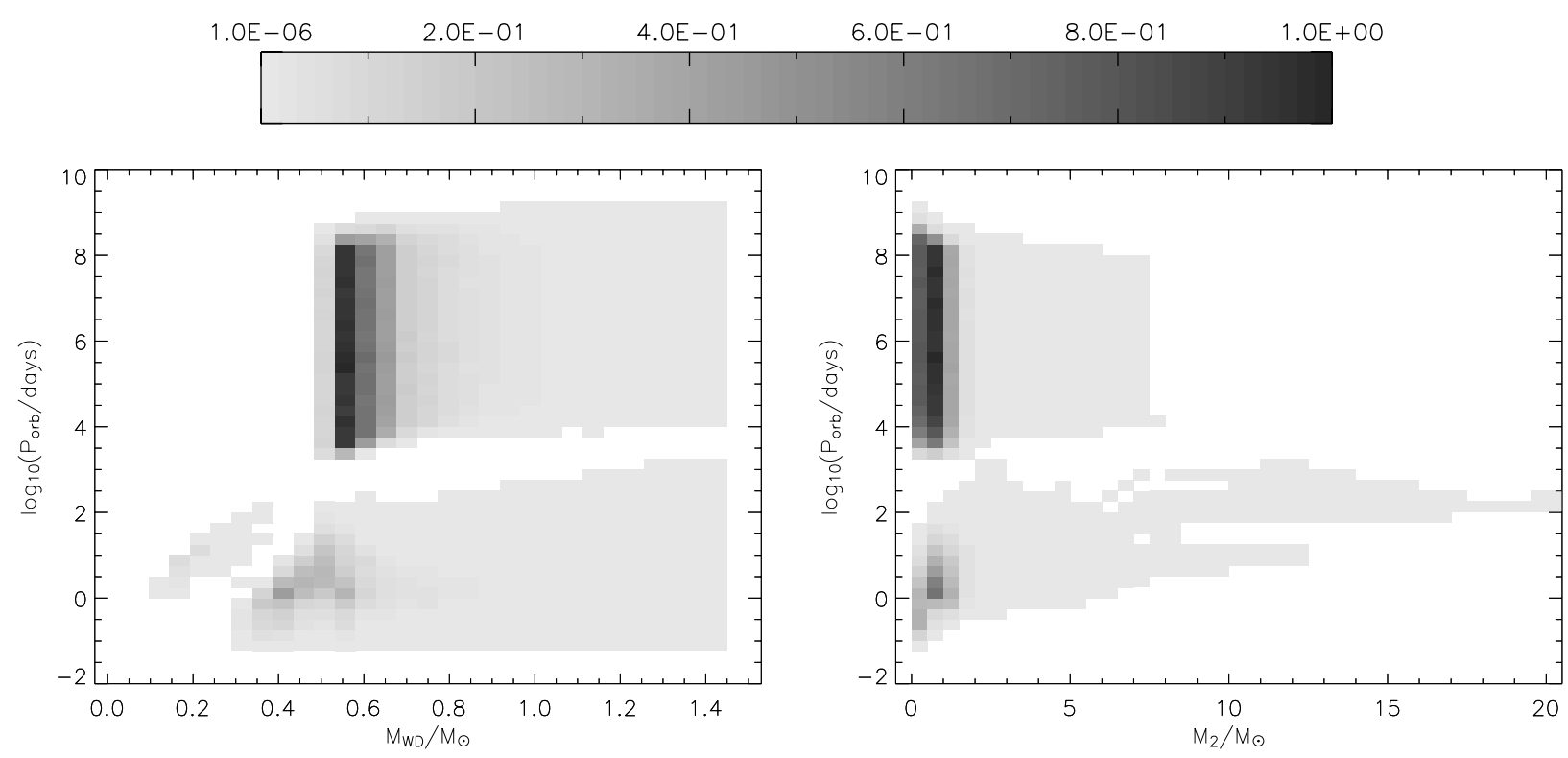

Fig. 9. Distribution of present-day WDMS binaries in the $\left(M_{\mathrm{WD}}, P_{\mathrm{orb}}\right)$ - and $\left(M_{2}, P_{\mathrm{orb}}\right)$-planes at the start of the WDMS binary phase.
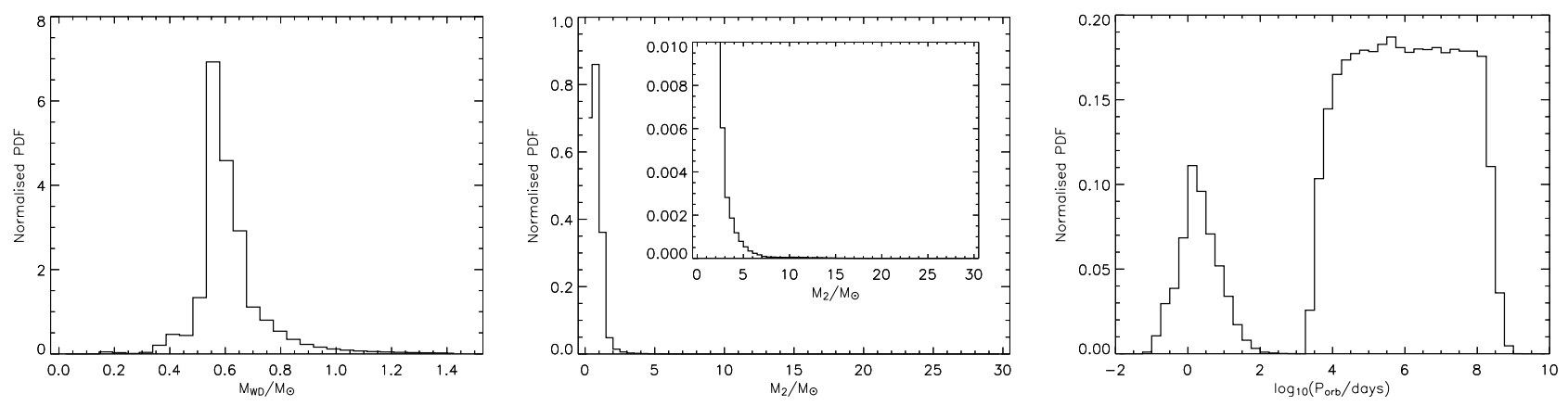

Fig. 10. White dwarf mass, secondary mass, and orbital period distributions of present-day WDMS binaries at the start of the WDMS binary phase. The distributions are normalised so that the integral over all systems found is equal to one.

Table 1. Population synthesis model parameters.

\begin{tabular}{lcc}
\hline \hline Model & $\gamma_{\text {RLOF }}$ & $\alpha_{\mathrm{CE}}$ \\
\hline A & Eq. (2) & 1.0 \\
G0 & 0.0 & 1.0 \\
G025 & 0.25 & 1.0 \\
G05 & 0.5 & 1.0 \\
G075 & 0.75 & 1.0 \\
G1 & 1.0 & 1.0 \\
CE1 & Eq. (2) & 0.2 \\
CE3 & Eq. (2) & 0.6 \\
CE6 & Eq. (2) & 3.0 \\
CE8 & Eq. (2) & 5.0 \\
\hline
\end{tabular}

and $P_{\text {orb }}$ we neglect the deviations of the high-density regions in the parameter space from a simple "rectangular" shape.

Since the mass-accretion parameter $\gamma_{\text {RLOF }}$ determines the amount of mass and thus the amount of orbital angular momentum lost from the system during episodes of dynamically stable Roche-lobe overflow, the parameter directly affects the evolution of WDMS binaries forming through formation channels 1-3. The parameter also indirectly affects the formation of WDMS binaries through channels 4-6 because it enters the stability criterion separating dynamically stable from dynamically unstable Roche-lobe overflow systems (for details see, e.g., the appendix in Kolb et al. 2001). The dependency of the criterion on $\gamma_{\text {RLOF }}$ is such than an increase in $\gamma_{\text {RLOF }}$ generally increases the critical mass ratio $Q_{\mathrm{c}}=M_{\text {donor }} / M_{\text {accretor }}$ separating dynamically stable $\left(Q<Q_{\mathrm{c}}\right)$ from dynamically unstable $\left(Q>Q_{\mathrm{c}}\right)$ Roche-lobe overflowing systems ${ }^{3}$. As we will see, the overall effect of the change in the stability criterion on the population is, however, rather small.

From Table 2, it follows that the parameter space occupied by the bulk of the WDMS binaries and their progenitors is fairly robust to changes in the mass-accretion param-

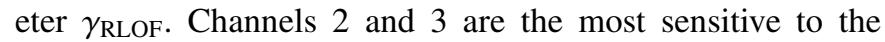
value of the mass-accretion parameter. The most obvious overall effect of changing $\gamma_{\text {RLOF }}$ is the decrease of the secondary mass $M_{2}$ at the birth of the WDMS binaries with increasing degree of non-conservativeness. In addition, for a given initial primary mass $M_{1}$, the increase of the critical mass ratio $Q_{\mathrm{c}}$

\footnotetext{
${ }^{3}$ Note that the definition of the mass ratio as $Q=M_{\text {donor }} / M_{\text {accretor }}$ may differ from the definition of the initial mass ratio given by $q=$ $M_{2} / M_{1}$.
} 
Table 2. The effect of the population synthesis model parameters on the masses and orbital periods of WDMS binary progenitors at the start of their evolution and of WDMS binaries at the time of their formation. For each formation channel, the mass and period ranges refer to bins containing at least $\sim 1 \%$ of the channel's contribution to the WDMS binary population. The quoted ranges should therefore be considered as approximate ranges for the bulk of the systems and not as strict limits. For the construction of the table, only binaries contributing to the present-day Galactic population are taken into account. Details of the parameters adopted in the different population synthesis models are listed in Table 1.

\begin{tabular}{|c|c|c|c|c|c|c|c|c|}
\hline \multirow[b]{2}{*}{ Channel } & \multirow[b]{2}{*}{ Mode } & & \multicolumn{3}{|c|}{ Initial parameters } & \multicolumn{3}{|c|}{ Formation parameters } \\
\hline & & & $M_{1} / M_{\odot}$ & $M_{2} / M_{\odot}$ & $P_{\text {orb }} /$ days & $M_{\mathrm{WD}} / M_{\odot}$ & $M_{2} / M_{\odot}$ & $P_{\text {orb }} /$ days \\
\hline \multirow[t]{4}{*}{1} & $\mathrm{~A}$ & (standard) & $1-3$ & $<2$ & $0.5-3$ & $0.15-0.35$ & $<5$ & $1-100$ \\
\hline & G0 & $\left(\gamma_{\mathrm{RLOF}}=0\right)$ & $1-3$ & $<2$ & $0.5-3$ & $0.15-0.35$ & $1-5$ & $3-100$ \\
\hline & G05 & $\left(\gamma_{\mathrm{RLOF}}=0.5\right)$ & $1-3$ & $<2$ & $0.5-3$ & $0.15-0.35$ & $<4$ & $2-100$ \\
\hline & G1 & $\left(\gamma_{\mathrm{RLOF}}=1\right)$ & $1-3$ & $<2$ & $0.5-3$ & $0.15-0.35$ & $<3$ & $1-100$ \\
\hline \multirow[t]{4}{*}{2} & $\mathrm{~A}$ & (standard) & $2-11$ & $1-9$ & $2-100$ & $0.3-1.44$ & $1-17$ & $10-1000$ \\
\hline & G0 & $\left(\gamma_{\mathrm{RLOF}}=0\right)$ & $4-11$ & $2-9$ & $2-100$ & $0.7-1.44$ & $6-17$ & $30-1000$ \\
\hline & G05 & $\left(\gamma_{\mathrm{RLOF}}=0.5\right)$ & $2-10$ & $1-8$ & $2-100$ & $0.3-1.44$ & $2-11$ & $20-1000$ \\
\hline & G1 & $\left(\gamma_{\mathrm{RLOF}}=1\right)$ & $2-9$ & $1-6$ & $2-100$ & $0.3-1.25$ & $1-6$ & $10-1000$ \\
\hline \multirow[t]{4}{*}{3} & $\mathrm{~A}$ & (standard) & $1-8$ & $1-7$ & $600-6000$ & $0.55-1.44$ & $1-7$ & $2000-20000$ \\
\hline & G0 & $\left(\gamma_{\mathrm{RLOF}}=0\right)$ & $1-8$ & $1-7$ & $600-6000$ & $0.55-1.44$ & $1-7$ & $2000-20000$ \\
\hline & G05 & $\left(\gamma_{\mathrm{RLOF}}=0.5\right)$ & $1-8$ & $<6$ & $600-6000$ & $0.5-1.4$ & $<6$ & $1000-20000$ \\
\hline & G1 & $\left(\gamma_{\mathrm{RLOF}}=1\right)$ & $1-6$ & $<4$ & $200-6000$ & $0.5-0.95$ & $<4$ & 600-20000 \\
\hline \multirow[t]{6}{*}{4} & CE1 & $\left(\alpha_{\mathrm{CE}}=0.2\right)$ & $1-2$ & $<2$ & $200-600$ & $0.4-0.5$ & $<2$ & $0.1-3$ \\
\hline & A & (standard) & $1-2$ & $<2$ & $30-600$ & $0.3-0.5$ & $<2$ & $0.1-20$ \\
\hline & CE8 & $\left(\alpha_{\mathrm{CE}}=5.0\right)$ & $1-2$ & $<2$ & $10-600$ & $0.25-0.5$ & $<2$ & $0.1-60$ \\
\hline & G0 & $\left(\gamma_{\mathrm{RLOF}}=0\right)$ & $1-2$ & $<2$ & $30-600$ & $0.3-0.5$ & $<2$ & $0.1-20$ \\
\hline & G05 & $\left(\gamma_{\mathrm{RLOF}}=0.5\right)$ & $1-2$ & $<2$ & $30-600$ & $0.3-0.5$ & $<2$ & $0.1-20$ \\
\hline & G1 & $\left(\gamma_{\mathrm{RLOF}}=1\right)$ & $1-2$ & $<2$ & $30-600$ & $0.3-0.5$ & $<2$ & $0.1-20$ \\
\hline \multirow[t]{6}{*}{5} & CE1 & $\left(\alpha_{\mathrm{CE}}=0.2\right)$ & $8-11$ & $4-7$ & $30-100$ & $0.95-1.4$ & $7-11$ & $2-6$ \\
\hline & A & (standard) & $5-11$ & $2-9$ & $20-1000$ & $0.75-1.44$ & $3-11$ & $0.5-20$ \\
\hline & CE8 & $\left(\alpha_{\mathrm{CE}}=5.0\right)$ & $3-11$ & $<7$ & $10-1000$ & $0.4-1.44$ & $<9$ & $0.3-30$ \\
\hline & G0 & $\left(\gamma_{\mathrm{RLOF}}=0\right)$ & $6-11$ & $2-9$ & $20-1000$ & $0.8-1.44$ & $3-11$ & $1-20$ \\
\hline & G05 & $\left(\gamma_{\mathrm{RLOF}}=0.5\right)$ & $4-11$ & $2-9$ & $20-1000$ & $0.7-1.44$ & $2-10$ & $0.5-20$ \\
\hline & G1 & $\left(\gamma_{\mathrm{RLOF}}=1\right)$ & $6-11$ & $2-9$ & $200-1000$ & $0.8-1.44$ & $2-9$ & $1-20$ \\
\hline \multirow[t]{6}{*}{$\overline{6}$} & CE1 & $\left(\alpha_{\mathrm{CE}}=0.2\right)$ & $1-2$ & $<3$ & $300-2000$ & $0.5-0.8$ & $<3$ & $0.1-10$ \\
\hline & A & (standard) & $1-5$ & $<3$ & $100-2000$ & $0.5-0.9$ & $<3$ & $0.1-60$ \\
\hline & CE8 & $\left(\alpha_{\mathrm{CE}}=5.0\right)$ & $1-5$ & $<3$ & $30-2000$ & $0.5-0.9$ & $<3$ & $0.2-300$ \\
\hline & G0 & $\left(\gamma_{\mathrm{RLOF}}=0\right)$ & $1-5$ & $<3$ & $100-2000$ & $0.5-0.9$ & $<3$ & $0.1-60$ \\
\hline & G05 & $\left(\gamma_{\mathrm{RLOF}}=0.5\right)$ & $1-5$ & $<3$ & $100-2000$ & $0.5-0.9$ & $<3$ & $0.1-60$ \\
\hline & G1 & $\left(\gamma_{\mathrm{RLOF}}=1\right)$ & $1-5$ & $<3$ & $100-2000$ & $0.5-0.9$ & $<3$ & $0.1-30$ \\
\hline 7 & $\mathrm{~A}$ & (standard) & $1-5$ & $<2$ & $10^{3}-10^{8}$ & $0.5-0.9$ & $<2$ & $3 \times 10^{3}-5 \times 10^{8}$ \\
\hline
\end{tabular}

with increasing values of $\gamma_{\text {RLOF }}$ implies that in models G05 and G1 lower initial secondary masses become available for dynamically stable Roche-lobe overflow. Due to the shape of the initial mass function (Eq. (3)) and the adopted initial mass ratio distribution (Eq. (4)), this behaviour shifts the bulk of the systems evolving through formation channels 2 and 3 towards lower primary and secondary masses. An increase in $\gamma_{\mathrm{RLOF}}$ is usually also accompanied by a decrease of the minimum orbital period at the birth of the WDMS binaries because more angular momentum is lost from the system. The stellar masses and orbital periods resulting from fully conservative mass-transfer (model G0) are furthermore very close to those found in our standard model (model A). This concordance arises from the small values of $\gamma_{\mathrm{RLOF}}$ inferred from Eq. (2) for binaries in which the thermal time scale of the accretor is not too much longer than the mass-transfer time scale of the donor. Finally, we note that the systems evolving through formation channels 1-3 have typical orbital periods longer than 1 day, in agreement with the orbital period distribution of post-mode II WDMS binaries derived by de Kool \& Ritter (1993). We recall that the terminology of mode II mass transfer was introduced by Webbink (1979) to indicate mass transfer from donor stars with radiative envelopes.

The common-envelope ejection efficiency $\alpha_{\mathrm{CE}}$ only affects the WDMS binaries forming through evolutionary channels 4-6. Since smaller values of $\alpha_{\mathrm{CE}}$ require more orbital energy to expel the envelope from the system, the minimum orbital period required for a binary to survive the spiral-in process increases with decreasing values of $\alpha_{\mathrm{CE}}$. For a given donor star mass $M_{1}$, the donor star in a binary surviving the commonenvelope phase therefore has more time to evolve when $\alpha_{\mathrm{CE}}$ is small, so that the minimum mass of the nascent white dwarf also increases with decreasing values of $\alpha_{\mathrm{CE}}$. Larger values of $\alpha_{\mathrm{CE}}$, on the other hand, yield wider orbital separations at the end of the common-envelope phase, making it easier for shortperiod binaries to survive the spiral-in process. These tendencies are clearly seen in Table 2 when comparing the variations 
in the parameter space between models CE1 $\left(\alpha_{\mathrm{CE}}=0.2\right), \mathrm{A}$ $\left(\alpha_{\mathrm{CE}}=1.0\right)$, and CE8 $\left(\alpha_{\mathrm{CE}}=5.0\right)$.

For a given a given orbital separation $a_{\mathrm{i}}$ at the onset of the common-envelope phase and a given donor star mass $M_{1}$, the orbital separation $a_{\mathrm{f}}$ at the end of the common-envelope phase furthermore increases with increasing secondary masses $M_{2}$. The mass of the secondary may therefore be the deciding factor determining whether or not a binary close to the borderline separating merging from non-merging systems survives the common-envelope phase or not. From Eq. (1), it follows that the minimum secondary mass $M_{2}$ required to survive the common-envelope phase increases with decreasing values of $\alpha_{\mathrm{CE}}$. This behaviour is responsible for the variations in the initial secondary mass range of binaries evolving through formation channel 5. Since $M_{1} / M_{2}>Q_{\mathrm{c}}$ is a necessary requirement for the occurrence of a common-envelope phase initiated by the primary, the changes in the initial secondary mass range are accompanied by similar changes in the initial primary mass range.

The influence of the donor star's mass $M_{1}$ on the outcome of the common-envelope phase can be understood by noting that the radius of giant-type stars is predominantly determined by the mass of their core, and that the radius of the star at the onset of Roche-lobe overflow is approximately equal to the radius of their Roche lobe. For a given initial orbital separation $a_{\mathrm{i}}$ and a given secondary mass $M_{2}$, the orbital separation $a_{\mathrm{f}}$ at the end of the common-envelope phase therefore increases with decreasing values of $M_{1}$. Consequently, the maximum primary mass $M_{1}$ for which a system may survive the common-envelope phase and the maximum mass of the resulting white dwarf decrease with decreasing values of $\alpha_{\mathrm{CE}}$. This behaviour is responsible for the lower upper limit on the initial primary mass range of formation channel 6 when $\alpha_{\mathrm{CE}}=0.2$ (model CE1).

Besides these general tendencies, the following additional differences between the various models may be observed upon inspection of the individual formation channels:

- Channel 2. - In model G0, the minimum initial primary mass $M_{1}$ increases from $2 M_{\odot}$ to $4 M_{\odot}$. The increase is associated with the destabilising effect of smaller $\gamma_{\mathrm{RLOF}}$-values on the mode of mass transfer from the primary: since the mainsequence radius of a star increases with increasing mass, a lower mass star has more time to evolve and thus to develop a deep convective envelope before it fills its Roche-lobe than a higher mass star in a binary with the same orbital period. For $\gamma_{\mathrm{RLOF}}=0$, primaries with mass $M_{1} \lesssim 4 M_{\odot}$ therefore lead to dynamically unstable mass transfer which usually results in the merger of the primary's core with its main-sequence companion. We furthermore note that for model A a gap occurs in the initial primary mass range between $3 M_{\odot}$ and $4 M_{\odot}$, in the white dwarf mass range at the time of formation between $0.4 M_{\odot}$ and $0.7 M_{\odot}$, and in the secondary mass range at the time of formation between $3 M_{\odot}$ and $6 M_{\odot}$. This behaviour is caused by the dependency of $\gamma_{\mathrm{RLOF}}$ on the ratio of the mass-transfer time scale of the donor to the thermal time scale of the accretor (see Eq. (2)). As a consequence, some combinations of masses and orbital periods in model A are subjected to the same destabilising effect of small $\gamma_{\mathrm{RLOF}}$-values as described for model G0.
Finally, we point that models A, G05 and G1 yield C/O white dwarfs with masses as low as $\sim 0.3 M_{\odot}$. Comparably low-mass C/O white dwarfs were also found by Iben \& Tutukov (1985) and Han et al. (2000) on the basis of more detailed numerical calculations.

- Channel 3. - In model G1, the minimum initial period of WDMS binary progenitors is significantly shorter than in any of the other population synthesis models considered. The reason for this is that in models A, G0, and G05, systems with initial orbital periods shorter than $\sim 600$ days undergo a commonenvelope phase instead of a dynamically stable Roche-lobe overflow phase on the first giant branch, so that they evolve through formation channel 4 or 5 rather than through formation channel 3 . When mass transfer becomes highly nonconservative, as in model G1, the mass-transfer phase becomes dynamically stable and the primary can avoid losing its entire envelope until it reaches the AGB.

- Channel 5. - In model G05, the minimum initial primary mass $M_{1}$ is about $2 M_{\odot}$ lower than in models G0 and G1. This behaviour is again associated with the stabilising effect of larger $\gamma_{\mathrm{RLOF}}$-values. Binaries with primary masses near $4 M_{\odot}$ do not survive the common-envelope phase in model G0, while they undergo a dynamically stable mass-transfer phase in model G1. In the latter case, the systems therefore evolve through formation channel 2 rather than through formation channel 5. When $\gamma_{\mathrm{RLOF}} \approx 0.5$ (model G05), mass transfer from primaries with $M_{1} \approx 4 M_{\odot}$ initially takes place on the thermal time scale of the donor star, so that the dynamical instability is delayed until the donor reaches the red-giant branch. Contrary to the outcome of the common-envelope phase in model G0, the decrease of the donor star's mass during the initial thermal time scale mass-transfer phase here yields a primary mass at the onset of the common-envelope phase that allows the binary to survive the spiral-in process. For a somewhat related reason, the longest possible initial orbital period decreases from $\sim 1000$ days to $\sim 100$ days in model CE1. As discussed in Sect. 3.2.2, systems with $P_{\text {orb }} \lesssim 100$ days start mass transfer on the thermal time scale of the donor star, while systems with $P_{\text {orb }} \gtrsim 100$ days go straight into the common-envelope phase. When $\alpha_{\mathrm{CE}}=0.2$, the primary masses of the latter systems at the onset of the common-envelope phase are too high to avoid a merger during the spiral-in process, so that only the group with $P_{\text {orb }} \lesssim 100$ days survives. Finally, the increase of the lower limit on the initial orbital periods in model G1 is associated with the disappearance of binaries with Hertzsprunggap donor stars (i.e. binaries with $P_{\text {orb }} \lesssim 200$ days). For highly non-conservative mass transfer these binaries evolve through formation channel 2 instead of through formation channel 5.

- Channel 6. - In model G1, the upper limit on the orbital periods at the formation time of the WDMS binaries decreases with respect to models G0 and G05. The stabilising effect of larger $\gamma_{\mathrm{RLOF}}$-values here implies that less mass needs to be lost via the stellar wind in order for mass transfer to be dynamically stable. Many of the longer period systems (i.e. the systems that have evolved furthest on the AGB and have thus lost the most mass in a stellar wind) therefore now evolve through formation channel 3 instead of formation channel 6. 


\section{WDMS binary numbers and formation rates}

Similar to the variations in the parameter space discussed in the previous section, the assumptions adopted in the various population synthesis models listed in Table 1 may affect the formation rates and the number of WDMS binaries currently populating the Galaxy. These variations do not apply to systems evolving through formation channel 7 since their evolution is governed solely by mass-loss and mass-accretion from stellar winds.

The total number of WDMS binaries currently populating the Galaxy and the relative number of systems that formed through the interacting and the non-interacting formation channels are listed in Table 3 for different initial mass ratio or initial secondary mass distributions. The most striking effect is the strong decrease of the absolute number of systems for the initial mass ratio distribution $n(q) \propto q^{-0.99}$, for $0<q \leq 1$. The decrease is related to the larger number of systems undergoing dynamically unstable mass transfer which ends in the merger of the two component stars.

Although the absolute number of systems resulting from formation channel 7 is independent of the adopted population synthesis model parameters, the relative number of systems changes due to variations in the number of systems forming through formation channels $1-6$. The non-interacting systems generally account for $75 \%$ to $85 \%$ of the total population if the initial mass ratio is distributed according to $n(q)=1$ or $n(q) \propto q$, for $0<q \leq 1$. This relative number increases to $95 \%$ when $\alpha_{\mathrm{CE}}=0.2$ (model CE1) due to the significant decrease of the number of systems surviving the common-envelope phase in formation channels 4-6. In the cases where the initial mass ratio is distributed according to $n(q) \propto q^{-0.99}$, for $0<q \leq 1$, or where the initial secondary mass $M_{2}$ is distributed independently from the primary mass $M_{1}$ according to the initial mass function given by Eq. (3), the relative contribution of the noninteracting systems to the population of WDMS binaries may be even larger.

In Table 4, the contribution of WDMS binaries with interacting progenitors to the present-day Galactic population is further subdivided according to the followed formation channel. The absolute number of systems forming through formation channels 1-6 increases with increasing values of $\gamma_{\text {RLOF, }}$ although the overall difference is rather small: the number increases by less than a factor of $\sim 1.25$ between models G0 $\left(\gamma_{\mathrm{RLOF}}=0\right)$ and G1 $\left(\gamma_{\mathrm{RLOF}}=1\right)$ for all initial mass ratio or initial secondary mass distributions considered. The effect of $\alpha_{\mathrm{CE}}$ on the absolute number of systems is larger: the number increases by about an order of magnitude between models CE1 $\left(\alpha_{\mathrm{CE}}=0.2\right)$ and CE8 $\left(\alpha_{\mathrm{CE}}=5.0\right)$. A similar dependency on $\alpha_{\mathrm{CE}}$ was noted by Iben et al. (1997) for WDMS binaries with secondaries less massive than $0.3 M_{\odot}$.

The relative contributions of formation channels 1-3 increase with increasing values of $\gamma_{\text {RLOF }}$ due to the stabilising effect of non-conservative mass transfer. Correspondingly, the relative contributions of channels 4 and 6 decrease with increasing values of $\gamma_{\text {RLOF }}$. The influence of $\gamma_{\text {RLOF }}$ is largest for formation channels 1-3 whose relative contributions increase by factors of $\sim 5-15$ between models G0 $\left(\gamma_{\mathrm{RLOF}}=0\right)$ and G1
Table 3. Total number of WDMS binaries currently populating the Galaxy and relative contributions of the interacting and the noninteracting formation channels for different initial mass ratio or initial secondary mass distributions. The absolute numbers may be converted into an approximate local space density of WDMS binaries by dividing them by $5 \times 10^{11} \mathrm{pc}^{3}$ (see Sect. 2.2).

\begin{tabular}{|c|c|c|c|}
\hline Model & $\begin{array}{c}\text { Total number } \\
\text { (Channel 1-7) }\end{array}$ & $\begin{array}{c}\text { Interacting } \\
(\text { Channel 1-6) }\end{array}$ & $\begin{array}{c}\text { Non-interacting } \\
\text { (Channel 7) }\end{array}$ \\
\hline \multicolumn{4}{|c|}{$n(q)=1,0<q \leq 1$} \\
\hline $\mathrm{A}$ & $1.6 \times 10^{9}$ & $13.6 \%$ & $86.4 \%$ \\
\hline G0 & $1.6 \times 10^{9}$ & $13.4 \%$ & $86.6 \%$ \\
\hline G025 & $1.6 \times 10^{9}$ & $13.7 \%$ & $86.3 \%$ \\
\hline G05 & $1.6 \times 10^{9}$ & $14.0 \%$ & $86.0 \%$ \\
\hline G075 & $1.6 \times 10^{9}$ & $14.6 \%$ & $85.4 \%$ \\
\hline G1 & $1.7 \times 10^{9}$ & $15.5 \%$ & $84.5 \%$ \\
\hline CE1 & $1.5 \times 10^{9}$ & $3.5 \%$ & $96.5 \%$ \\
\hline CE3 & $1.6 \times 10^{9}$ & $9.8 \%$ & $90.2 \%$ \\
\hline CE6 & $1.8 \times 10^{9}$ & $20.1 \%$ & $79.2 \%$ \\
\hline CE8 & $1.8 \times 10^{9}$ & $23.4 \%$ & $76.6 \%$ \\
\hline \multicolumn{4}{|c|}{ 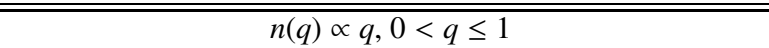 } \\
\hline $\mathrm{A}$ & $1.3 \times 10^{9}$ & $15.6 \%$ & $84.4 \%$ \\
\hline G0 & $1.3 \times 10^{9}$ & $15.4 \%$ & $84.6 \%$ \\
\hline G025 & $1.3 \times 10^{9}$ & $15.8 \%$ & $84.2 \%$ \\
\hline G05 & $1.3 \times 10^{9}$ & $16.2 \%$ & $83.8 \%$ \\
\hline G075 & $1.3 \times 10^{9}$ & $17.2 \%$ & $82.8 \%$ \\
\hline G1 & $1.3 \times 10^{9}$ & $18.5 \%$ & $81.5 \%$ \\
\hline CE1 & $1.2 \times 10^{9}$ & $4.9 \%$ & $95.1 \%$ \\
\hline CE3 & $1.2 \times 10^{9}$ & $11.9 \%$ & $88.1 \%$ \\
\hline CE6 & $1.4 \times 10^{9}$ & $22.4 \%$ & $77.6 \%$ \\
\hline CE8 & $1.5 \times 10^{9}$ & $24.7 \%$ & $75.3 \%$ \\
\hline \multicolumn{4}{|c|}{ 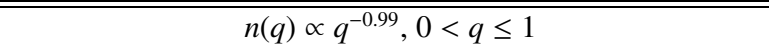 } \\
\hline $\mathrm{A}$ & $6.6 \times 10^{7}$ & $10.7 \%$ & $89.3 \%$ \\
\hline G0 & $6.6 \times 10^{7}$ & $10.6 \%$ & $89.4 \%$ \\
\hline G025 & $6.6 \times 10^{7}$ & $10.7 \%$ & $89.3 \%$ \\
\hline G05 & $6.7 \times 10^{7}$ & $10.9 \%$ & $89.1 \%$ \\
\hline G075 & $6.7 \times 10^{7}$ & $11.2 \%$ & $88.8 \%$ \\
\hline G1 & $6.7 \times 10^{7}$ & $11.5 \%$ & $88.5 \%$ \\
\hline CE1 & $6.0 \times 10^{7}$ & $2.0 \%$ & $98.0 \%$ \\
\hline CE3 & $6.4 \times 10^{7}$ & $7.0 \%$ & $93.0 \%$ \\
\hline CE6 & $7.3 \times 10^{7}$ & $18.6 \%$ & $81.4 \%$ \\
\hline CE8 & $7.6 \times 10^{7}$ & $21.6 \%$ & $78.4 \%$ \\
\hline \multicolumn{4}{|c|}{$M_{2}$ from IMF given by Eq. (3) } \\
\hline $\mathrm{A}$ & $2.4 \times 10^{9}$ & $10.3 \%$ & $89.7 \%$ \\
\hline G0 & $2.4 \times 10^{9}$ & $10.1 \%$ & $89.9 \%$ \\
\hline G025 & $2.4 \times 10^{9}$ & $10.3 \%$ & $89.7 \%$ \\
\hline G05 & $2.4 \times 10^{9}$ & $10.4 \%$ & $89.6 \%$ \\
\hline G075 & $2.4 \times 10^{9}$ & $10.6 \%$ & $89.4 \%$ \\
\hline G1 & $2.4 \times 10^{9}$ & $10.7 \%$ & $89.3 \%$ \\
\hline CE1 & $2.2 \times 10^{9}$ & $1.8 \%$ & $98.2 \%$ \\
\hline CE3 & $2.3 \times 10^{9}$ & $6.6 \%$ & $93.4 \%$ \\
\hline CE6 & $2.6 \times 10^{9}$ & $18.2 \%$ & $81.8 \%$ \\
\hline CE8 & $2.7 \times 10^{9}$ & $21.3 \%$ & $78.7 \%$ \\
\hline
\end{tabular}

$\left(\gamma_{\mathrm{RLOF}}=1\right)$. Increasing the common-envelope ejection efficiency $\alpha_{\mathrm{CE}}$, on the other hand, increases the relative contributions of channels 4 and 5 . The increase is caused by the larger number of systems surviving the common-envelope phase resulting from case B Roche-lobe overflow events. The relative contribution of formation channel 6, somewhat surprisingly, 
Table 4. Total number of present-day Galactic WDMS binaries that formed through formation channels 1-6 and relative contributions of each formation channel for different initial mass ratio or initial secondary mass distributions. The absolute numbers may be converted into an approximate local space density by dividing them by $5 \times 10^{11} \mathrm{pc}^{3}$.

\begin{tabular}{|c|c|c|c|c|c|c|c|}
\hline \multirow[b]{3}{*}{ Model } & \multirow[b]{3}{*}{ Total number } & \multicolumn{3}{|c|}{ " DYNAMICALLY STABLE MASS TRANSFER } & \multicolumn{3}{|c|}{ "COMMON - ENVELOPE PHASE } \\
\hline & & \multicolumn{2}{|c|}{$\begin{array}{c}\swarrow \\
\text { CASE B }\end{array}$} & \multirow{2}{*}{$\begin{array}{c}\searrow \\
\text { Case C } \\
\downarrow \\
\text { NHE STAR } \\
\text { (Channel 3) } \\
\end{array}$} & \multicolumn{2}{|c|}{$\stackrel{\swarrow}{\swarrow}$} & \multirow{2}{*}{$\begin{array}{c}\searrow \\
\text { CASE C } \\
\downarrow \\
\text { WD oR NHE STAR } \\
(\text { Channel 6) }\end{array}$} \\
\hline & & $\begin{array}{c}\swarrow \\
\text { WD } \\
(\text { Channel 1) }\end{array}$ & $\begin{array}{c}\searrow \\
\text { NHE STAR } \\
\text { (Channel 2) }\end{array}$ & & $\begin{array}{c}\swarrow \\
\text { WD } \\
\text { (Channel 4) }\end{array}$ & $\begin{array}{c}\searrow \\
\text { NHE STAR } \\
\text { (Channel 5) }\end{array}$ & \\
\hline \multicolumn{8}{|c|}{$n(q)=1,0<q \leq 1$} \\
\hline $\mathrm{A}$ & $2.2 \times 10^{8}$ & $3.4 \%$ & $0.2 \%$ & $0.1 \%$ & $40.5 \%$ & $0.1 \%$ & $55.7 \%$ \\
\hline G0 & $2.2 \times 10^{8}$ & $2.0 \%$ & $0.2 \%$ & $0.1 \%$ & $41.1 \%$ & $0.1 \%$ & $56.5 \%$ \\
\hline G025 & $2.2 \times 10^{8}$ & $4.1 \%$ & $0.3 \%$ & $0.2 \%$ & $40.1 \%$ & $0.2 \%$ & $55.1 \%$ \\
\hline G05 & $2.3 \times 10^{8}$ & $6.2 \%$ & $0.7 \%$ & $0.4 \%$ & $39.1 \%$ & $0.2 \%$ & $53.5 \%$ \\
\hline G075 & $2.4 \times 10^{8}$ & $10.2 \%$ & $1.6 \%$ & $0.6 \%$ & $37.1 \%$ & $0.1 \%$ & $50.5 \%$ \\
\hline G1 & $2.6 \times 10^{8}$ & $14.8 \%$ & $2.8 \%$ & $1.5 \%$ & $34.4 \%$ & $0.1 \%$ & $46.5 \%$ \\
\hline CE1 & $5.2 \times 10^{7}$ & $14.5 \%$ & $1.0 \%$ & $0.4 \%$ & $18.8 \%$ & $<0.1 \%$ & $65.2 \%$ \\
\hline CE3 & $1.5 \times 10^{8}$ & $4.9 \%$ & $0.3 \%$ & $0.1 \%$ & $35.5 \%$ & $0.1 \%$ & $59.0 \%$ \\
\hline CE6 & $3.7 \times 10^{8}$ & $2.0 \%$ & $0.1 \%$ & $0.1 \%$ & $47.1 \%$ & $0.4 \%$ & $50.2 \%$ \\
\hline CE8 & $4.3 \times 10^{8}$ & $1.8 \%$ & $0.1 \%$ & $0.1 \%$ & $49.7 \%$ & $0.8 \%$ & $47.6 \%$ \\
\hline \multicolumn{8}{|c|}{$\bar{n} n(q) \propto q, 0<q \leq 1$} \\
\hline $\mathrm{A}$ & $2.0 \times 10^{8}$ & $3.8 \%$ & $0.3 \%$ & $0.2 \%$ & $44.9 \%$ & $0.2 \%$ & $50.6 \%$ \\
\hline G0 & $2.0 \times 10^{8}$ & $2.7 \%$ & $0.3 \%$ & $0.2 \%$ & $45.4 \%$ & $0.2 \%$ & $51.2 \%$ \\
\hline G025 & $2.1 \times 10^{8}$ & $5.2 \%$ & $0.5 \%$ & $0.4 \%$ & $44.2 \%$ & $0.2 \%$ & $49.6 \%$ \\
\hline G05 & $2.1 \times 10^{8}$ & $7.7 \%$ & $1.0 \%$ & $0.6 \%$ & $42.8 \%$ & $0.2 \%$ & $47.8 \%$ \\
\hline G075 & $2.3 \times 10^{8}$ & $12.7 \%$ & $2.2 \%$ & $1.1 \%$ & $39.9 \%$ & $0.1 \%$ & $44.1 \%$ \\
\hline G1 & $2.5 \times 10^{8}$ & $18.6 \%$ & $3.6 \%$ & $2.6 \%$ & $36.0 \%$ & $0.1 \%$ & $39.3 \%$ \\
\hline CE1 & $5.7 \times 10^{7}$ & $13.6 \%$ & $1.2 \%$ & $0.6 \%$ & $20.2 \%$ & $<0.1 \%$ & $64.3 \%$ \\
\hline CE3 & $1.5 \times 10^{8}$ & $5.2 \%$ & $0.5 \%$ & $0.2 \%$ & $39.1 \%$ & $0.1 \%$ & $54.9 \%$ \\
\hline CE6 & $3.2 \times 10^{8}$ & $2.4 \%$ & $0.2 \%$ & $0.1 \%$ & $52.6 \%$ & $0.5 \%$ & $44.1 \%$ \\
\hline CE8 & $3.6 \times 10^{8}$ & $2.2 \%$ & $0.2 \%$ & $0.1 \%$ & $55.6 \%$ & $0.9 \%$ & $41.1 \%$ \\
\hline \multicolumn{8}{|c|}{$n(q) \propto q^{-0.99}, 0<q \leq 1$} \\
\hline $\mathrm{A}$ & $7.1 \times 10^{6}$ & $2.3 \%$ & $0.1 \%$ & $<0.1 \%$ & $35.5 \%$ & $0.1 \%$ & $62.0 \%$ \\
\hline G0 & $7.0 \times 10^{6}$ & $1.1 \%$ & $0.1 \%$ & $<0.1 \%$ & $35.9 \%$ & $0.1 \%$ & $62.7 \%$ \\
\hline G025 & $7.1 \times 10^{6}$ & $2.5 \%$ & $0.2 \%$ & $0.1 \%$ & $35.4 \%$ & $0.1 \%$ & $61.8 \%$ \\
\hline G05 & $7.2 \times 10^{6}$ & $3.8 \%$ & $0.4 \%$ & $0.1 \%$ & $34.9 \%$ & $0.1 \%$ & $60.8 \%$ \\
\hline G075 & $7.5 \times 10^{6}$ & $6.0 \%$ & $0.8 \%$ & $0.2 \%$ & $33.9 \%$ & $<0.1 \%$ & $59.0 \%$ \\
\hline G1 & $7.7 \times 10^{6}$ & $8.6 \%$ & $1.6 \%$ & $0.6 \%$ & $32.6 \%$ & $<0.1 \%$ & $56.6 \%$ \\
\hline CE1 & $1.2 \times 10^{6}$ & $13.3 \%$ & $0.7 \%$ & $0.2 \%$ & $17.9 \%$ & $<0.1 \%$ & $67.9 \%$ \\
\hline CE3 & $4.5 \times 10^{6}$ & $3.6 \%$ & $0.2 \%$ & $0.1 \%$ & $32.0 \%$ & $<0.1 \%$ & $64.1 \%$ \\
\hline CE6 & $1.4 \times 10^{7}$ & $1.2 \%$ & $0.1 \%$ & $<0.1 \%$ & $39.8 \%$ & $0.2 \%$ & $58.8 \%$ \\
\hline CE8 & $1.6 \times 10^{7}$ & $1.0 \%$ & $0.1 \%$ & $<0.1 \%$ & $41.5 \%$ & $0.6 \%$ & $56.9 \%$ \\
\hline \multicolumn{8}{|c|}{ 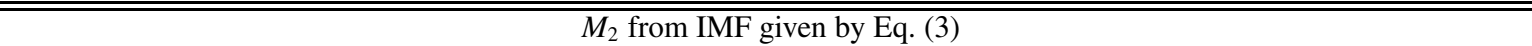 } \\
\hline $\mathrm{A}$ & $2.5 \times 10^{8}$ & $2.3 \%$ & $<0.1 \%$ & $<0.1 \%$ & $37.0 \%$ & $<0.1 \%$ & $60.7 \%$ \\
\hline G0 & $2.4 \times 10^{8}$ & $1.0 \%$ & $<0.1 \%$ & $<0.1 \%$ & $37.5 \%$ & $<0.1 \%$ & $61.5 \%$ \\
\hline G025 & $2.5 \times 10^{8}$ & $2.3 \%$ & $<0.1 \%$ & $<0.1 \%$ & $37.0 \%$ & $<0.1 \%$ & $60.7 \%$ \\
\hline G05 & $2.5 \times 10^{8}$ & $3.5 \%$ & $0.1 \%$ & $0.1 \%$ & $36.5 \%$ & $<0.1 \%$ & $59.9 \%$ \\
\hline G075 & $2.6 \times 10^{8}$ & $5.4 \%$ & $0.2 \%$ & $0.1 \%$ & $35.7 \%$ & $<0.1 \%$ & $58.6 \%$ \\
\hline G1 & $2.6 \times 10^{8}$ & $6.9 \%$ & $0.4 \%$ & $0.3 \%$ & $35.0 \%$ & $<0.1 \%$ & $57.4 \%$ \\
\hline CE1 & $4.1 \times 10^{7}$ & $14.0 \%$ & $0.1 \%$ & $<0.1 \%$ & $19.0 \%$ & $<0.1 \%$ & $66.9 \%$ \\
\hline CE3 & $1.5 \times 10^{8}$ & $3.7 \%$ & $<0.1 \%$ & $<0.1 \%$ & $33.5 \%$ & $<0.1 \%$ & $62.8 \%$ \\
\hline CE6 & $4.8 \times 10^{8}$ & $1.2 \%$ & $<0.1 \%$ & $<0.1 \%$ & $40.9 \%$ & $<0.1 \%$ & $57.8 \%$ \\
\hline CE8 & $5.8 \times 10^{8}$ & $1.0 \%$ & $<0.1 \%$ & $<0.1 \%$ & $42.6 \%$ & $0.2 \%$ & $56.2 \%$ \\
\hline
\end{tabular}

decreases with increasing values of $\alpha_{\mathrm{CE}}$, but the absolute number of systems forming through this channel still increases. The decrease of the relative number is therefore caused by the more prominent increase of the absolute number of systems forming through channels 4 and 5 . For similar reasons, the relative contributions of channels 1-3 also decrease with increasing values of $\alpha_{\mathrm{CE}}$.

The population of WDMS binaries with interacting progenitors is dominated by systems forming through formation channels 4 and 6. Depending on the adopted model 

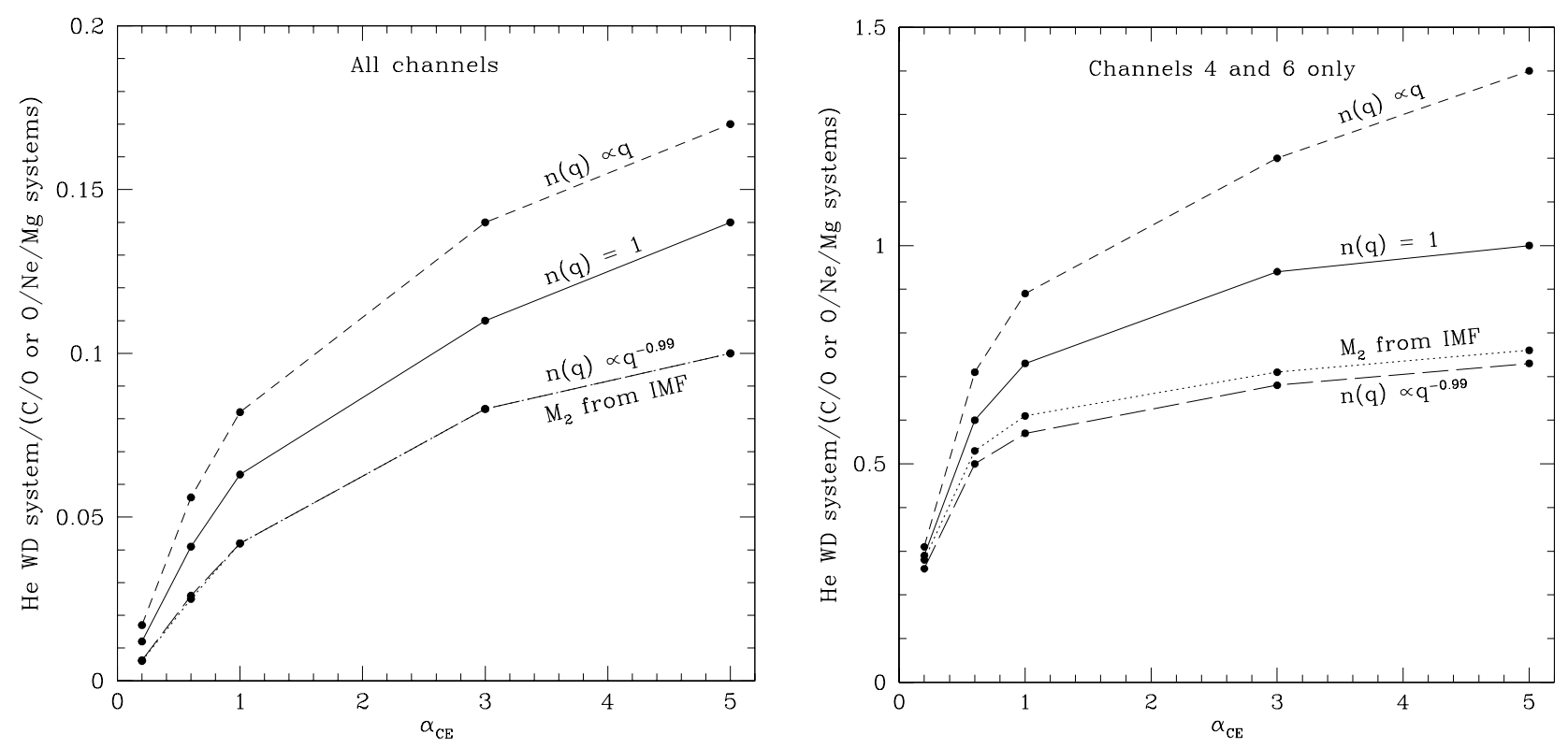

Fig. 11. Ratio of He white dwarf WDMS binaries to $\mathrm{C} / \mathrm{O}$ or $\mathrm{O} / \mathrm{Ne} / \mathrm{Mg}$ WDMS binaries for different initial mass ratio or initial secondary mass distributions. The left-hand panel shows the ratios as a function of the common-envelope ejection efficiency $\alpha_{\mathrm{CE}}$ for WDMS binaries forming through all considered formation channels, while the right-hand panel shows the ratios for systems forming through formation channels 4 and 6 only. In the left-hand panel, the curves associated with $n(q) \propto q^{-0.99}$ and with $M_{2}$ drawn independently from the same IMF as $M_{1}$ are almost indistinguishable.

parameters, they generally account for $80 \%$ to $95 \%$ of the population if the initial mass ratio is distributed according to $n(q)=1$ or $n(q) \propto q$, for $0<q \leq 1$, or even for $90 \%$ to $99 \%$ of the population if the initial mass ratio is distributed according to $n(q) \propto q^{-0.99}$, for $0<q \leq 1$, or the initial secondary mass is distributed according to the initial mass function given by Eq. (3). The short-period binaries resulting from channels 4 and 6 (see Table 2) together with the long-period systems forming from wide non-interacting progenitors are therefore responsible for the bimodal nature of the orbital period distribution shown in Fig. 10. The smallest contributions to the population of WDMS binaries with interacting progenitors stem from formation channels 2, 3 and 5. We note, however, that channels 2 and 5 are interesting candidates for the formation of WD + early B-star binaries with long and short orbital periods, respectively (Fig. 3). We will address these channels in this context in a forthcoming paper (Willems et al., in preparation).

In Table 5, we list the present-day birthrates of WDMS binaries forming through each of the considered formation channels. The birthrate of systems forming through formation channel 7 is independent of the adopted population synthesis model parameters and amounts to $\sim 0.3 \mathrm{yr}^{-1}$, unless the initial mass ratio is distributed according to $n(q) \propto q^{-0.99}$, for $0<q \leq 1$. In the latter case, the birthrate of wide non-interacting systems is of the order of $0.01 \mathrm{yr}^{-1}$. The birthrate derived for our standard model with $n(q)=1$, for $0<q \leq 1$, is in excellent agreement with the birthrate for $\mathrm{C} / \mathrm{O}$ white dwarfs in wide binaries of $0.29 \mathrm{yr}^{-1}$ derived by Iben \& Tutukov (1986b).

The general dependencies of the birthrates of formation channels 1-6 on the adopted population synthesis model are similar to those of the absolute and relative numbers of systems described above. The effect of changing $\gamma_{\text {RLOF }}$ is largest for channels 2 and 3 where the birthrate increases by an order of magnitude between models G0 $\left(\gamma_{\mathrm{RLOF}}=0\right)$ and G1 $\left(\gamma_{\text {RLOF }}=1\right)$. The impact of $\gamma_{\text {RLOF }}$ on the other channels is typically smaller than a factor of $\sim 3$. Changes in $\alpha_{\mathrm{CE}}$, on the other hand, predominantly affect formation channels 4 and 5 . The birthrates of systems forming through these channels increase by 1 and 3 orders of magnitude, respectively, between model CE1 $\left(\alpha_{\mathrm{CE}}=0.2\right)$ and model CE8 $\left(\alpha_{\mathrm{CE}}=5.0\right)$. In the case where the initial secondary mass is distributed according to the same initial mass function as the initial primary mass, the birthrate of channel 5 increases by 4 orders of magnitude. The birthrate of systems forming through formation channel 6 varies by less than a factor of $\sim 5$, while the birthrates of systems forming through formation channels 1-3 are not affected at all. For $\gamma_{\mathrm{RLOF}} \lessgtr 0.25$ and $\alpha_{\mathrm{CE}} \gtrsim 0.6$, the total birthrate of all WDMS binaries forming through a common-envelope phase (channels 4-6) is furthermore about 10 times larger than the total birthrate of all WDMS binaries forming through a stable Roche-lobe overflow phase (channels 1-3). This is again in excellent agreement with de Kool \& Ritter (1993).

For conclusion, we consider the ratio of the number of systems containing a He white dwarf to the number of systems containing a $\mathrm{C} / \mathrm{O}$ or $\mathrm{O} / \mathrm{Ne} / \mathrm{Mg}$ white dwarf. This ratio is independent of the normalisation of the adopted star formation rate (Eq. (6)) and of the fraction of stars in binaries. Due to the dominance of the wide non-interacting systems and the systems forming through the common-envelope channels 4 and 6 , the ratio is fairly insensitive to changes in the mass-

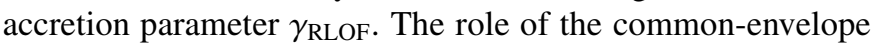
ejection parameter $\alpha_{\mathrm{CE}}$, on the other hand, is much more prominent. This is illustrated in the left-hand panel of Fig. 11. For $\alpha_{\mathrm{CE}}=0.2$ (model CE1) the ratio takes comparably small values 
Table 5. Birthrates of Galactic WDMS binaries forming through formation channels $1-7$ in the case of different initial mass ratio or initial secondary mass distributions. The rates may be converted into approximate local birthrates by dividing them by $5 \times 10^{11} \mathrm{pc}^{3}$.

\begin{tabular}{|c|c|c|c|c|c|c|c|}
\hline \multirow[b]{3}{*}{ Model } & \multicolumn{3}{|c|}{ DYNAMICALLY STABLE MASS TRANSFER } & \multicolumn{3}{|c|}{ COMMON - ENVELOPE PHASE } & \multirow{3}{*}{$\begin{array}{c}\text { Non- } \\
\text { INTERACTING } \\
\text { SysteMS } \\
\text { (Channel 7) }\end{array}$} \\
\hline & \multicolumn{2}{|c|}{ CASE B } & \multirow{2}{*}{$\begin{array}{c}\searrow \\
\text { Case C } \\
\downarrow \\
\text { NHE STAR } \\
\text { (Channel 3) } \\
\end{array}$} & \multicolumn{2}{|c|}{$\begin{array}{c}\swarrow \\
\text { CASE B }\end{array}$} & \multirow{2}{*}{$\begin{array}{c}\searrow \\
\text { CASE C } \\
\downarrow \\
\text { WD oR NHE STAR } \\
(\text { Channel 6) }\end{array}$} & \\
\hline & $\begin{array}{c}\swarrow \\
\text { WD } \\
(\text { Channel 1) }\end{array}$ & $\begin{array}{c}\searrow \\
\text { NHE STAR } \\
\text { (Channel 2) }\end{array}$ & & $\begin{array}{c}\swarrow \\
\text { WD } \\
\text { (Channel 4) }\end{array}$ & $\begin{array}{c}\searrow \\
\text { NHE STAR } \\
\text { (Channel 5) }\end{array}$ & & \\
\hline \multicolumn{8}{|c|}{$n(q)=1,0<q \leq 1$} \\
\hline $\mathrm{A}$ & $5.0 \times 10^{-3}$ & $1.0 \times 10^{-3}$ & $3.5 \times 10^{-4}$ & $3.5 \times 10^{-2}$ & $5.2 \times 10^{-4}$ & $3.4 \times 10^{-2}$ & $3.0 \times 10^{-1}$ \\
\hline G0 & $4.6 \times 10^{-3}$ & $9.6 \times 10^{-4}$ & $3.5 \times 10^{-4}$ & $3.5 \times 10^{-2}$ & $5.1 \times 10^{-4}$ & $3.4 \times 10^{-2}$ & $3.0 \times 10^{-1}$ \\
\hline G025 & $6.8 \times 10^{-3}$ & $1.5 \times 10^{-3}$ & $5.9 \times 10^{-4}$ & $3.5 \times 10^{-2}$ & $6.5 \times 10^{-4}$ & $3.4 \times 10^{-2}$ & $3.0 \times 10^{-1}$ \\
\hline G05 & $8.0 \times 10^{-3}$ & $3.1 \times 10^{-3}$ & $8.8 \times 10^{-4}$ & $3.5 \times 10^{-2}$ & $7.4 \times 10^{-4}$ & $3.4 \times 10^{-2}$ & $3.0 \times 10^{-1}$ \\
\hline G075 & $1.0 \times 10^{-2}$ & $6.2 \times 10^{-3}$ & $1.3 \times 10^{-3}$ & $3.5 \times 10^{-2}$ & $2.5 \times 10^{-4}$ & $3.3 \times 10^{-2}$ & $3.0 \times 10^{-1}$ \\
\hline G1 & $1.2 \times 10^{-2}$ & $7.5 \times 10^{-3}$ & $2.7 \times 10^{-3}$ & $3.5 \times 10^{-2}$ & $2.6 \times 10^{-4}$ & $3.2 \times 10^{-2}$ & $3.0 \times 10^{-1}$ \\
\hline CE1 & $5.0 \times 10^{-3}$ & $1.0 \times 10^{-3}$ & $3.5 \times 10^{-4}$ & $8.3 \times 10^{-3}$ & $2.9 \times 10^{-6}$ & $1.3 \times 10^{-2}$ & $3.0 \times 10^{-1}$ \\
\hline CE3 & $5.0 \times 10^{-3}$ & $1.0 \times 10^{-3}$ & $3.5 \times 10^{-4}$ & $2.6 \times 10^{-2}$ & $1.8 \times 10^{-4}$ & $2.7 \times 10^{-2}$ & $3.0 \times 10^{-1}$ \\
\hline CE6 & $5.0 \times 10^{-3}$ & $1.0 \times 10^{-3}$ & $3.5 \times 10^{-4}$ & $5.6 \times 10^{-2}$ & $2.4 \times 10^{-3}$ & $4.4 \times 10^{-2}$ & $3.0 \times 10^{-1}$ \\
\hline CE8 & $5.0 \times 10^{-3}$ & $1.0 \times 10^{-3}$ & $3.5 \times 10^{-4}$ & $6.5 \times 10^{-2}$ & $4.3 \times 10^{-3}$ & $4.4 \times 10^{-2}$ & $3.0 \times 10^{-1}$ \\
\hline \multicolumn{8}{|c|}{$\overline{c n(q) \propto q, 0<q \leq 1}$} \\
\hline $\mathrm{A}$ & $6.6 \times 10^{-3}$ & $1.4 \times 10^{-3}$ & $5.6 \times 10^{-4}$ & $3.8 \times 10^{-2}$ & $6.6 \times 10^{-4}$ & $3.5 \times 10^{-2}$ & $3.0 \times 10^{-1}$ \\
\hline G0 & $6.3 \times 10^{-3}$ & $1.3 \times 10^{-3}$ & $5.6 \times 10^{-4}$ & $3.8 \times 10^{-2}$ & $6.4 \times 10^{-4}$ & $3.5 \times 10^{-2}$ & $3.0 \times 10^{-1}$ \\
\hline G025 & $9.1 \times 10^{-3}$ & $2.0 \times 10^{-3}$ & $9.8 \times 10^{-4}$ & $3.8 \times 10^{-2}$ & $8.0 \times 10^{-4}$ & $3.5 \times 10^{-2}$ & $3.0 \times 10^{-1}$ \\
\hline G05 & $1.1 \times 10^{-2}$ & $4.0 \times 10^{-3}$ & $1.4 \times 10^{-3}$ & $3.8 \times 10^{-2}$ & $9.1 \times 10^{-4}$ & $3.4 \times 10^{-2}$ & $3.0 \times 10^{-1}$ \\
\hline G075 & $1.4 \times 10^{-2}$ & $8.3 \times 10^{-3}$ & $2.2 \times 10^{-3}$ & $3.8 \times 10^{-2}$ & $2.9 \times 10^{-4}$ & $3.3 \times 10^{-2}$ & $3.0 \times 10^{-1}$ \\
\hline G1 & $1.5 \times 10^{-2}$ & $1.0 \times 10^{-2}$ & $4.6 \times 10^{-3}$ & $3.7 \times 10^{-2}$ & $2.9 \times 10^{-4}$ & $3.2 \times 10^{-2}$ & $3.0 \times 10^{-1}$ \\
\hline CE1 & $6.6 \times 10^{-3}$ & $1.4 \times 10^{-3}$ & $5.6 \times 10^{-4}$ & $9.4 \times 10^{-3}$ & $3.2 \times 10^{-6}$ & $1.5 \times 10^{-2}$ & $3.0 \times 10^{-1}$ \\
\hline CE3 & $6.6 \times 10^{-3}$ & $1.4 \times 10^{-3}$ & $5.6 \times 10^{-4}$ & $2.8 \times 10^{-2}$ & $2.3 \times 10^{-4}$ & $2.9 \times 10^{-2}$ & $3.0 \times 10^{-1}$ \\
\hline CE6 & $6.6 \times 10^{-3}$ & $1.4 \times 10^{-3}$ & $5.6 \times 10^{-4}$ & $5.9 \times 10^{-2}$ & $2.8 \times 10^{-3}$ & $4.4 \times 10^{-2}$ & $3.0 \times 10^{-1}$ \\
\hline CE8 & $6.6 \times 10^{-3}$ & $1.4 \times 10^{-3}$ & $5.6 \times 10^{-4}$ & $6.8 \times 10^{-2}$ & $4.7 \times 10^{-3}$ & $4.4 \times 10^{-2}$ & $3.0 \times 10^{-1}$ \\
\hline \multicolumn{8}{|c|}{$\bar{n} n(q) \propto q^{-0.99}, 0<q \leq 1$} \\
\hline $\mathrm{A}$ & $8.3 \times 10^{-5}$ & $1.5 \times 10^{-5}$ & $4.5 \times 10^{-6}$ & $9.2 \times 10^{-4}$ & $8.8 \times 10^{-6}$ & $1.0 \times 10^{-3}$ & $9.8 \times 10^{-3}$ \\
\hline G0 & $7.3 \times 10^{-5}$ & $1.4 \times 10^{-5}$ & $4.5 \times 10^{-6}$ & $9.2 \times 10^{-4}$ & $8.5 \times 10^{-6}$ & $1.0 \times 10^{-3}$ & $9.8 \times 10^{-3}$ \\
\hline G025 & $1.1 \times 10^{-4}$ & $2.2 \times 10^{-5}$ & $7.4 \times 10^{-6}$ & $9.2 \times 10^{-4}$ & $1.1 \times 10^{-5}$ & $1.0 \times 10^{-3}$ & $9.8 \times 10^{-3}$ \\
\hline G05 & $1.3 \times 10^{-4}$ & $4.9 \times 10^{-5}$ & $1.1 \times 10^{-5}$ & $9.2 \times 10^{-4}$ & $1.3 \times 10^{-5}$ & $1.0 \times 10^{-3}$ & $9.8 \times 10^{-3}$ \\
\hline G075 & $1.7 \times 10^{-4}$ & $9.5 \times 10^{-5}$ & $1.6 \times 10^{-5}$ & $9.2 \times 10^{-4}$ & $4.9 \times 10^{-6}$ & $1.0 \times 10^{-3}$ & $9.8 \times 10^{-3}$ \\
\hline G1 & $1.8 \times 10^{-4}$ & $1.2 \times 10^{-4}$ & $3.3 \times 10^{-5}$ & $9.2 \times 10^{-4}$ & $5.0 \times 10^{-6}$ & $9.9 \times 10^{-4}$ & $9.8 \times 10^{-3}$ \\
\hline CE1 & $8.3 \times 10^{-5}$ & $1.5 \times 10^{-5}$ & $4.5 \times 10^{-6}$ & $1.8 \times 10^{-4}$ & $5.0 \times 10^{-8}$ & $3.3 \times 10^{-4}$ & $9.8 \times 10^{-3}$ \\
\hline CE3 & $8.3 \times 10^{-5}$ & $1.5 \times 10^{-5}$ & $4.5 \times 10^{-6}$ & $6.5 \times 10^{-4}$ & $2.9 \times 10^{-6}$ & $7.8 \times 10^{-4}$ & $9.8 \times 10^{-3}$ \\
\hline CE6 & $8.3 \times 10^{-5}$ & $1.5 \times 10^{-5}$ & $4.5 \times 10^{-6}$ & $1.5 \times 10^{-3}$ & $4.5 \times 10^{-5}$ & $1.4 \times 10^{-3}$ & $9.8 \times 10^{-3}$ \\
\hline CE8 & $8.3 \times 10^{-5}$ & $1.5 \times 10^{-5}$ & $4.5 \times 10^{-6}$ & $1.8 \times 10^{-3}$ & $9.0 \times 10^{-5}$ & $1.5 \times 10^{-3}$ & $9.8 \times 10^{-3}$ \\
\hline \multicolumn{8}{|c|}{$M_{2}$ from IMF given by Eq. (3) } \\
\hline $\mathrm{A}$ & $2.3 \times 10^{-3}$ & $4.0 \times 10^{-5}$ & $2.0 \times 10^{-5}$ & $3.3 \times 10^{-2}$ & $1.7 \times 10^{-5}$ & $3.1 \times 10^{-2}$ & $3.3 \times 10^{-1}$ \\
\hline G0 & $1.9 \times 10^{-3}$ & $2.7 \times 10^{-5}$ & $2.0 \times 10^{-5}$ & $3.3 \times 10^{-2}$ & $1.5 \times 10^{-5}$ & $3.1 \times 10^{-2}$ & $3.3 \times 10^{-1}$ \\
\hline G025 & $3.0 \times 10^{-3}$ & $5.8 \times 10^{-5}$ & $6.0 \times 10^{-5}$ & $3.3 \times 10^{-2}$ & $2.7 \times 10^{-5}$ & $3.1 \times 10^{-2}$ & $3.3 \times 10^{-1}$ \\
\hline G05 & $3.3 \times 10^{-3}$ & $2.4 \times 10^{-4}$ & $1.2 \times 10^{-4}$ & $3.3 \times 10^{-2}$ & $3.4 \times 10^{-5}$ & $3.1 \times 10^{-2}$ & $3.3 \times 10^{-1}$ \\
\hline G075 & $4.1 \times 10^{-3}$ & $5.2 \times 10^{-4}$ & $2.0 \times 10^{-4}$ & $3.3 \times 10^{-2}$ & $1.1 \times 10^{-5}$ & $3.0 \times 10^{-2}$ & $3.3 \times 10^{-1}$ \\
\hline G1 & $4.3 \times 10^{-3}$ & $6.7 \times 10^{-4}$ & $5.2 \times 10^{-4}$ & $3.3 \times 10^{-2}$ & $1.2 \times 10^{-5}$ & $3.0 \times 10^{-2}$ & $3.3 \times 10^{-1}$ \\
\hline CE1 & $2.3 \times 10^{-3}$ & $4.0 \times 10^{-5}$ & $2.0 \times 10^{-5}$ & $6.7 \times 10^{-3}$ & $6.2 \times 10^{-8}$ & $1.0 \times 10^{-2}$ & $3.3 \times 10^{-1}$ \\
\hline CE3 & $2.3 \times 10^{-3}$ & $4.0 \times 10^{-5}$ & $2.0 \times 10^{-5}$ & $2.3 \times 10^{-2}$ & $4.5 \times 10^{-6}$ & $2.4 \times 10^{-2}$ & $3.3 \times 10^{-1}$ \\
\hline CE6 & $2.3 \times 10^{-3}$ & $4.0 \times 10^{-5}$ & $2.0 \times 10^{-5}$ & $5.5 \times 10^{-2}$ & $2.0 \times 10^{-4}$ & $4.2 \times 10^{-2}$ & $3.3 \times 10^{-1}$ \\
\hline CE8 & $2.3 \times 10^{-3}$ & $4.0 \times 10^{-5}$ & $2.0 \times 10^{-5}$ & $6.5 \times 10^{-2}$ & $7.7 \times 10^{-4}$ & $4.5 \times 10^{-2}$ & $3.3 \times 10^{-1}$ \\
\hline
\end{tabular}

for each of the initial mass ratio or initial secondary mass distributions considered. Provided that a complete observational sample of WDMS binaries can be compiled and the nature of the white dwarf can be established, comparison of the observationally derived ratio of $\mathrm{He}$ white dwarf systems to $\mathrm{C} / \mathrm{O}$ or $\mathrm{O} / \mathrm{Ne} / \mathrm{Mg}$ white dwarf systems with the theoretically predicted values may therefore constrain the common-envelope ejection efficiency. For higher values of $\alpha_{\mathrm{CE}}$, the effect of changing $\alpha_{\mathrm{CE}}$ can not be distinguished from the effect of changing the adopted initial mass ratio or initial secondary mass distribution. As shown in the right-hand panel of Fig. 11, a similar dependency on $\gamma_{\mathrm{RLOF}}$ and $\alpha_{\mathrm{CE}}$ is also found when the subset of 

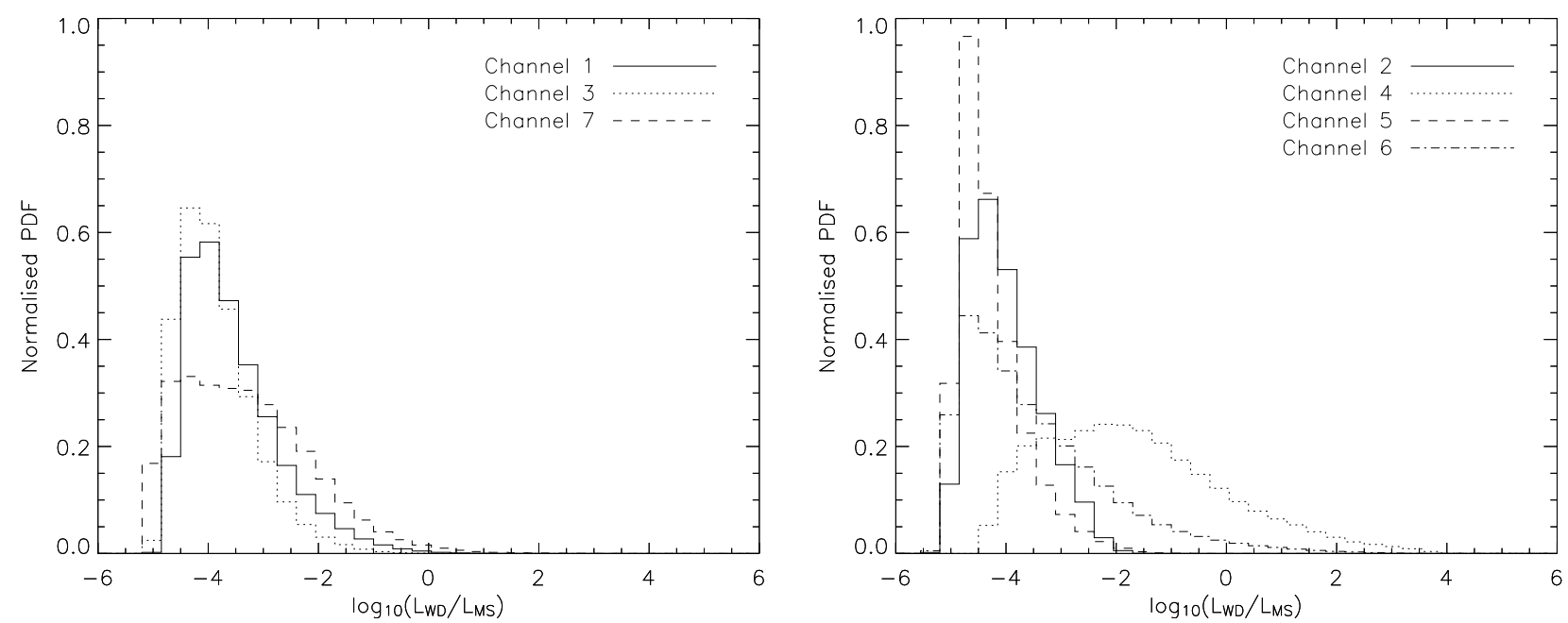

Fig. 12. Normalised distribution functions of white dwarf to main-sequence star luminosity ratios for formation channels $1-7$ in the case of population synthesis model A and the initial mass ratio distribution $n(q)=1$.

short-period systems resulting from the dominant channels 4 and 6 is considered. This subset is potentially more interesting than the whole sample of WDMS binaries since shorter period systems are easier to detect observationally than longer period systems.

\section{Luminosity ratios and radial velocities}

The detection and identification of WDMS binaries generally relies on the ability to detect the white dwarf's signature in the composite binary spectrum and to determine the components' orbital radial-velocity variations. Regardless of any additional selection effects, the detection of the white dwarf's spectral signature becomes increasingly difficult with decreasing ratio of the white dwarf's luminosity to the main-sequence star's luminosity, while the detection of radial-velocity variations becomes more difficult with increasing orbital periods.

The luminosity ratio may be particularly stringent for binaries with older white dwarfs since the time during which a white dwarf is detectable is typically of the order of $10^{8}$ years or shorter (e.g. Iben et al. 1997). The distribution of the luminosity ratios $L_{\mathrm{WD}} / L_{\mathrm{MS}}$, where $L_{\mathrm{WD}}$ is the luminosity of the white dwarf and $L_{\mathrm{MS}}$ that of its main-sequence companion, for WDMS binaries forming through the different formation channels are displayed in Fig. 12 in the case of population synthesis model $\mathrm{A}$ and the initial mass ratio distribution $n(q)=1$. For the determination of the luminosity ratios, we adopted the expression for white dwarf cooling by Hurley et al. (2000) and neglected the relatively small increase of the secondary's luminosity during its evolution on the main sequence. The luminosity ratio distributions typically peak between $10^{-5}$ and $10^{-3}$, except in the case of formation channel 4 where a much broader distribution is found which extends up to significantly higher luminosity ratios. The figure furthermore shows that channels 4 and 6 contain a significant number of WDMS binaries with small luminosity ratios, so that it may actually be hard to compile a complete sample of systems to constrain the commonenvelope ejection efficiency $\alpha_{\mathrm{CE}}$.
In Fig. 13, we combine the luminosity ratio distributions shown in Fig. 12 with the relative contributions of the different formation channels to the WDMS binary population given in Tables 3 and 4 to determine the fraction of systems for wich the white dwarf to main-sequence star luminosity ratio is larger than a given limiting ratio $\left(L_{\mathrm{WD}} / L_{\mathrm{MS}}\right)_{\text {crit }}$. The thick solid line represents the total for all systems forming through formation channels $1-7$, while the thin lines distinguish between the contributions of the individual formation channels. For $\left(L_{\mathrm{WD}} / L_{\mathrm{MS}}\right)_{\text {crit }}=10^{-6}$, the fractional contributions of the different channels correspond to those obtained from Tables 3 and 4 . The wide non-interacting systems dominate up to $\left(L_{\mathrm{WD}} / L_{\mathrm{MS}}\right)_{\text {crit }} \approx 10^{-1}$. For larger values of $\left(L_{\mathrm{WD}} / L_{\mathrm{MS}}\right)_{\text {crit }}$, the systems forming through formation channel 4 become equally important.

The amplitudes $K_{\mathrm{WD}}$ and $K_{\mathrm{MS}}$ of the white dwarf and the main-sequence star's orbital radial-velocity variations in the case of a typical orbital inclination $i=60^{\circ}$ and under the assumption of a fixed Keplerian orbit are listed in Table 6. This assumption breaks down for short-period systems in which magnetic braking and/or gravitational radiation cause the components to spiral-in towards each other. However, we do not expect this to significantly affect our radial-velocity estimates, unless the orbital evolution time scale becomes shorter than or comparable to the white dwarf cooling time. As presentday radial-velocity surveys easily reach accuracies down to $10 \mathrm{~km} \mathrm{~s}^{-1}$, all formation channels except channels 3 and 7 produce WDMS binaries with readily detectable radial-velocity amplitudes. If the spectra of the component stars can be disentangled, the common-envelope scenarios furthermore produce WDMS binaries in which both components may have observable radial-velocity variations.

\section{Concluding remarks}

We used the BiSEPS binary population synthesis code described by Willems \& Kolb (2002) to study the population of detached white dwarf main-sequence star binaries forming through seven distinct evolutionary channels. In six of these, 

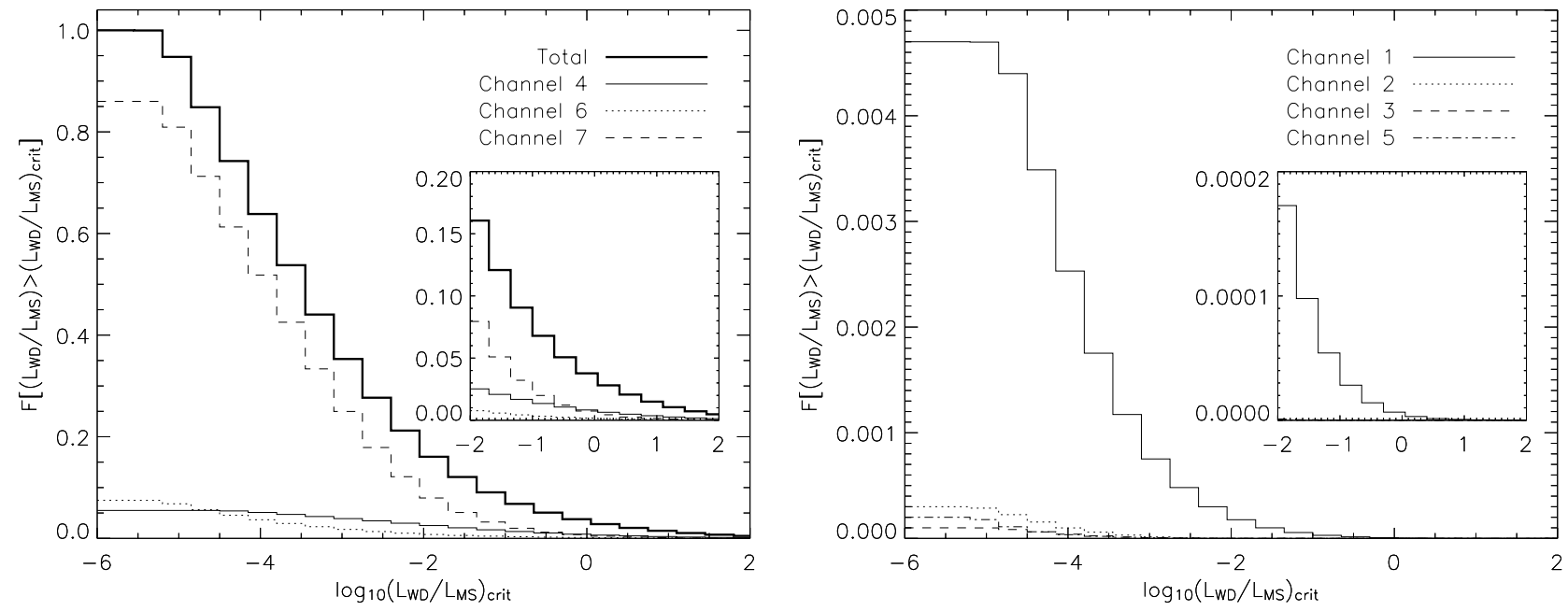

Fig. 13. The fraction $F$ of systems for wich the white dwarf to main-sequence star luminosity ratio is larger than the limiting ratio $\left(L_{\mathrm{WD}} / L_{\mathrm{MS}}\right)_{\text {crit }}$, in the case of population synthesis model A and the initial mass ratio distribution $n(q)=1$. The thick solid line represents the total for all systems forming through formation channels $1-7$, while the thin lines distinguish between the contributions of the different formation channels. The inserts zoom in on the regions where the distribution functions are small.

Table 6. Typical orbital radial-velocity amplitudes of WDMS binary components in the case of population synthesis model A and the initial mass ratio distribution $n(q)=1$. The radial-velocity amplitudes correspond to a typical orbital inclination of $60^{\circ}$.

\begin{tabular}{lrr}
\hline \hline Formation channel & \multicolumn{1}{c}{$K_{\mathrm{WD}}$} & \multicolumn{1}{c}{$K_{\mathrm{MS}}$} \\
\hline 1 (Stable case B RLOF $\rightarrow$ WD) & $80-100 \mathrm{~km} \mathrm{~s}^{-1}$ & $5-10 \mathrm{~km} \mathrm{~s}^{-1}$ \\
2 (Stable case B RLOF $\rightarrow$ nHe star) & $50-100 \mathrm{~km} \mathrm{~s}^{-1}$ & $5-10 \mathrm{~km} \mathrm{~s}^{-1}$ \\
3 (Stable case C RLOF $\rightarrow$ nHe star) & $5-15 \mathrm{~km} \mathrm{~s}^{-1}$ & $2-5 \mathrm{~km} \mathrm{~s}^{-1}$ \\
$4($ Case B CE $\rightarrow$ WD) & $70-220 \mathrm{~km} \mathrm{~s}^{-1}$ & $30-140 \mathrm{~km} \mathrm{~s}^{-1}$ \\
5 (Case B CE $\rightarrow \mathrm{nHe}$ star) & $170-220 \mathrm{~km} \mathrm{~s}^{-1}$ & $20-30 \mathrm{~km} \mathrm{~s}^{-1}$ \\
6 (Case C CE $\rightarrow$ nHe star or WD) & $40-170 \mathrm{~km} \mathrm{~s}^{-1}$ & $20-140 \mathrm{~km} \mathrm{~s}^{-1}$ \\
7 (Non-interacting systems) & $1-10 \mathrm{~km} \mathrm{~s}^{-1}$ & $1-5 \mathrm{~km} \mathrm{~s}^{-1}$ \\
\hline
\end{tabular}

the white dwarf is formed through binary interactions resulting from Roche-lobe overflow. The channels mainly differ in the stability and the outcome of the mass-transfer phase that eventually gives rise to the formation of the white dwarf. The six formation channels are depicted schematically in Figs. 1 and $4-8$. The seventh evolutionary channel is characterised by the absence of any type of binary interactions other than mass and angular momentum exchange via a stellar wind. Binaries evolving through this channel are typically very wide, so that the white dwarf forms in much the same way as it would if the primary were a single star.

Our results show that the wide non-interacting binaries generally comprise more than $75 \%$ of the total WDMS binary population. The remaining part of the population is dominated by systems undergoing a common-envelope phase when the white dwarf progenitor ascends the first giant branch or the asymptotic giant branch. The total number of systems currently populating the Galaxy, the relative number of systems evolving through each formation channel, and the birthrates of systems forming through each channel are given in Tables 3-5 for different different assumptions about the fate of the mass transferred during dynamically stable Roche-lobe overflow, different common-envelope ejection efficiencies, and different initial mass ratio or secondary mass distributions. The total number of systems is of the order of $\sim 10^{9}$ when a flat initial mass ratio distribution $n(q)=1$, for $0<q \leq 1$, is considered. This number decreases to $\sim 7 \times 10^{7}$ for an initial mass ratio distribution of the form $n(q) \propto q^{-0.99}$, for $0<q \leq 1$. An even stronger decrease is found for the birthrates of systems forming through the different formation channels. Besides the different assumptions regarding the treatment of dynamically stable and unstable mass transfer, the total number of WDMS binaries currently populating the Galaxy also depends on the finite life time of the systems which, in the case of short-period systems, may be affected by the adopted magnetic braking prescription. A detailed analysis of the effects of different braking laws is, however, beyond the scope of this investigation.

The total number of Galactic WDMS binaries evolving through a common-envelope phase is of the order of $\sim 2 \times 10^{8}$ when a flat initial mass ratio distribution $n(q)=1$ is considered, and of the order of $\sim 7 \times 10^{6}$ when an initial mass ratio distribution of the form $n(q) \propto q^{-0.99}$ is considered. Under the assumption that the Galaxy has an effective volume of $5 \times 10^{11} \mathrm{pc}^{3}$, these numbers correspond to approximate local space densities of $4 \times 10^{-4} \mathrm{pc}^{-3}$ and $10^{-5} \mathrm{pc}^{-3}$, respectively. The latter space density is comparable to the space density of $6-30 \times 10^{-6} \mathrm{pc}^{-3}$ derived by Schreiber \& Gänsicke (2003) from an observed sample of 30 post-common-envelope binaries with low-mass main-sequence secondaries. However, since the space densities derived by Schreiber \& Gänsicke (2003) are likely to be lower 
limits, the space density of $4 \times 10^{-4} \mathrm{pc}^{-3}$ is not necessarily in disagreement with observations. We also note that 12 of the 30 post-common-envelope binaries considered by Schreiber \& Gänsicke (2003) contain an sdOB primary instead of a white dwarf. These sdOB + main-sequence star systems are not included in our simulated WDMS binary samples.

We furthermore find that the ratio of the number of $\mathrm{He}$ white dwarf systems to the number of $\mathrm{C} / \mathrm{O}$ or $\mathrm{O} / \mathrm{Ne} / \mathrm{Mg}$ white dwarf systems is weakly dependent on the amount of mass lost from the system during dynamically stable Roche-lobe overflow and strongly dependent on the common-envelope ejection efficiency. We therefore propose that comparison of the observationally derived ratio from, e.g., large-scale planet-search mission as SuperWASP, COROT, and Kepler with the ratios presented in Fig. 11 may yield constraints on the commonenvelope ejection efficiency, provided that the efficiency is low. For higher efficiencies, the role of the ejection efficiency parameter cannot be distinguished from the role of the initial mass ratio or initial secondary mass distribution. We note, however, that the theoretically predicted ratios are derived under the assumption of a constant envelope binding-energy parameter and a constant envelope-ejection parameter. In addition, the derivation of constraints on the common-envelope ejection efficiency requires the detection of WDMS binaries with luminosity ratios down to $10^{-5}$, which poses a severe and not easily overcome observational challenge.

This paper is to serve as a first comprehensive step in a study of more specific subclasses of WDMS binaries and binaries descending from them. In future investigations we will address WDMS binaries in the context of pre-cataclysmic variables, double white dwarfs (Willems et al., in preparation), and white dwarf + B-star binaries (Willems et al., in preparation). Other potentially interesting applications are the study of type Ia supernovae progenitors through both the "standard" evolutionary channel and the recently proposed thermal time scale mass-transfer channel (King et al. 2003).

Acknowledgements. We are grateful to Jarrod Hurley, Onno Pols, and Chris Tout for sharing their SSE software package and to the referee, Gijs Nelemans, whose constructive report and valuable suggestions contributed to the improvement of the paper. Rob Mundin contributed to the subroutine that calculates the common-envelope evolution. This research was supported by the British Particle Physics and Astronomy Research Council (PPARC) and made use of NASA's Astrophysics Data System Bibliographic Services.

\section{References}

Boissier, S., \& Prantzos, N. 1999, MNRAS, 307, 857

Bond, H. E., \& Livio, M. 1990, ApJ, 355, 568

Burleigh, M. R., \& Barstow, M. A. 2000, A\&A, 359, 977

Burleigh, M. R., Barstow, M. A., Schenker, K. J., et al. 2001, MNRAS, 327,1158

Chen, X., \& Han, Z. 2002, MNRAS, 335, 948

Chen, X., \& Han, Z. 2003, MNRAS, 341, 662

de Kool, M. 1992, A\&A, 261, 188

de Kool, M., \& Ritter, H. 1993, A\&A, 267, 397

de Loore, C., \& Vanbeveren, D. 1995, A\&A, 304, 220

Farmer, A. J., \& Agol, E. 2003, ApJ, 592, 1151

Han, Z., Podsiadlowski, P., \& Eggleton, P. P. 1995, MNRAS, 272, 800
Han, Z., Tout, C. A., \& Eggleton, P. P. 2000, MNRAS, 319, 215

Hillwig, T. C., Honeycutt, R. K., \& Robertson, J. W. 2000, ApJ, 120, 1113

Hjellming, M. S. 1989, Rapid mass transfer in binary systems, Ph.D. Thesis Illinois University at Urbana-Champaign, Savoy

Holberg, J. B., Barstow, M. A., Bruhweiler, F. C., Cruise, A. M., \& Penny, A. J. 1998, ApJ, 497, 935

Hurley, J. R., Pols, O. R., \& Tout, C. A. 2000, MNRAS, 315, 543

Hurley, J. R., Tout, C. A., \& Pols, O. R. 2002, MNRAS, 329, 897

Iben, I. Jr., \& Tutukov, A. V. 1984, ApJS, 54, 335

Iben, I. Jr., \& Tutukov, A. V. 1985, ApJS, 58, 661

Iben, I. Jr., \& Tutukov, A. V. 1986a, ApJ, 311, 742

Iben, I. Jr., \& Tutukov, A. V. 1986b, ApJ, 311, 753

Iben, I. Jr., \& Tutukov, A. V. 1987, ApJ, 313, 727

Iben, I. Jr., Tutukov, A. V., \& Yungelson, L. R. 1997, ApJ, 475, 291

Joss, P. C., Rappaport, S., \& Lewis, W. 1987, ApJ, 319, 180

King, A. R., Rolfe, D. J., \& Schenker, K. 2003, MNRAS, 341, L35

Kolb, U., Rappaport, S., Schenker, K., \& Howell, S. 2001, ApJ, 563, 958

Kroupa, P. 2001, MNRAS, 322, 231

Kroupa, P., Tout, C. A., \& Gilmore, G. 1993, MNRAS, 262, 545

Langer, N., Deutschmann, A., Wellstein, S., \& Höflich, P. 2000, A\&A, 362, 1046

Law, W. Y., \& Ritter, H. 1983, A\&A, 123, 33

Livio, M. 1992, in Planetary Nebulae, ed. A. Acker, \& R. Weinberger (Dordrecht: Kluwer Academic Publishers), IAU Symp., 155, 279

Marsh, T. R., Dhillon, V. S., \& Duck, S. R. 1995, MNRAS, 275, 828

Marsh, T. R. 2000, New Astron. Rev., 44, 119

Maxted, P. F. L., Marsh, T. R., \& Moran, C. K. J. 2000, MNRAS, 319, 305

Nelson, C. A., \& Eggleton, P. P. 2001, ApJ, 552, 664

Politano, M. J. 1988, Ph.D. Thesis, University of Illinois

Politano, M. J. 1996, ApJ, 465, 338

Pols, O. R., Cote, J., Waters, L. B. F. M., \& Heise, J. 1991, A\&A, 241, 419

Pylyser, E., \& Savonije, G. J. 1988, A\&A, 191, 57

Pylyser, E., \& Savonije, G. J. 1989, A\&A, 208, 52

Rappaport, S., Podsiadlowski, Ph., Joss, P. C., di Stefano, R., \& Han, Z. 1995, MNRAS, 273, 731

Raymond, S. N., Szkody, P., Hawley, S. L., et al. 2003, AJ, 125, 2621

Ritter, H. 1999, MNRAS, 309, 360

Saffer, R. A., Livio, M., \& Yungelson, L. R. 1998, ApJ, 502, 394

Sandquist, E. L., Taam, R. E., \& Burkert, A. 2000, ApJ, 533, 984

Savonije, G. J. 1987, Nature, 325, 416

Scalo, J. 1998, The Stellar Initial Mass Function (38th Herstmonceux Conference), ASP Conf. Ser., 142, 201

Schreiber, M. R., \& Gänsicke, B. T. 2003, A\&A, 406, 305

Shobbrook, R. R., Wu, K., Soria, R., \& Johnston, H. M. 2003, A\&A, submitted

Silvestri, N. M., Oswalt, T. D., \& Hawley, S. L. 2002, AJ, 124, 1118

Tauris, T. M., \& Savonije, G. J. 1999, A\&A, 350, 928

van den Heuvel, E. P. J. 1981, in Fundamental Problems in the Theory of Stellar Evolution (Dordrecht: D. Reidel Publishing Co.), IAU Symp., 93, 155

van der Linden, T. J. 1987, A\&A, 178, 170

Vennes, S., Christian, D. J., \& Thorstensen, J. R. 1998, ApJ, 502, 763

Waters, L. B. F. M., Taylor, A. R., van den Heuvel, E. P. J., Habets, G. M. H. J., \& Persi, P. 1988, A\&A, 198, 200

Webbink, R. F. 1979, in White Dwarfs and Variable Degenerate Stars, ed. H. van Horn, \& V. Weidemann (University of Rochester Press), IAU Coll., 53, 426

Webbink, R. F. 1985, in Interacting Binary Stars, ed. J. E. Pringle, \& R. A. Wade (Cambridge: Cambridge University Press)

Webbink, R. F., Rappaport, S., \& Savonije, G. J. 1983, ApJ, 270, 678

Weidemann, V. 1990, ARA\&A, 28, 103

Willems, B., \& Kolb, U. 2002, MNRAS, 337, 1004 